\title{
Highlights
}

\section{On the structure of triangle-free 3-connected matroids}

Jaime C. dos Santos ${ }^{1}$

- Local characterization for 2-minor-irreducible matroids;

- One-to-one correspondence between diamantic and totally triangular matroids;

- Determine the 2-minor-irreducible binary matroids covered by emeralds.

\footnotetext{
${ }^{1}$ This research did not receive any specific grant from funding agencies in the public, commercial, or not-for-profit sectors.
} 


\title{
On the structure of triangle-free 3-connected matroids
}

\author{
Jaime C. dos Santos \\ Unidade Acadêmica do Cabo de Santo Agostinho \\ Universidade Federal Rural de Pernambuco \\ Cabo de Santo Agostinho, Pernambuco, 54518-430, Brazil
}

\begin{abstract}
Let $\mathcal{N}$ be an arbitrary class of matroids, closed under isomorphism. For $k$ a positive integer, we say that $M \in \mathcal{N}$ is $k$-minor-irreducible if $M$ has no minor $N \in \mathcal{N}$ such that $1 \leq|E(M)|-|E(N)| \leq k$. Tutte's Wheels and Whirls Theorem establish that, up to isomorphism, there are only two families of 1-minor-irreducible matroids in the class of 3-connected matroids. More recently, Lemos classified the 3-minor-irreducibles with at least 14 elements in the class of triangle-free 3 -connected matroids. Here we prove a local characterization for the 2-minor-irreducible matroids with at least 11 elements in the class of triangle-free 3-connected matroids. This local characterization is used to establish two new families of 2-minor-irreducible matroids in this class.
\end{abstract}

Keywords: Matroid, 3-connected, Minor, Irreducible, Triangle, Triad

\section{Introduction}

For arbitrary matroids $M$ and $N, N<M$ means that $N$ is isomorphic to a proper minor of $M$. As usual, $E(M)$ denotes the ground set of $M$ and $M \simeq N$ means that $M$ and $N$ are isomorphic matroids. In this paper, $\mathcal{F}$ denotes the class of triangle-free 3-connected matroids.

Take $M \in \mathcal{N}$, where $\mathcal{N}$ denotes an arbitrary class of matroids closed under isomorphism. We say that $M$ is $k$-minor-reducible in $\mathcal{N}$, for $k$ a positive integer, if $M$ has a minor $N \in \mathcal{N}$ such that $1 \leq|E(M)|-|E(N)| \leq k$.

Email address: jaime.cesar@ufrpe.br (Jaime C. dos Santos)

This research did not receive any specific grant from funding agencies in the public, commercial, or not-for-profit sectors. 
Otherwise, $M$ is said $k$-minor-irreducible in $\mathcal{N}$. Given a matroid $M \in \mathcal{N}$ and $k$ a positive integer, there is a finite sequence of matroids $M_{0}, M_{1}, \ldots, M_{l} \in$ $\mathcal{N}$, for $l \geq 0$, such that $M_{l}<M_{l-1}<\cdots<M_{1}<M_{0}$ with $M_{0}=M$, $1 \leq\left|E\left(M_{i}\right)\right|-\left|E\left(M_{i+1}\right)\right| \leq k$ for $i \in\{0,1, \ldots, l-1\}$ and $M_{l}$ is $k$-minorirreducible in $\mathcal{N}$.

Since our focus is on triangle-free 3-connected matroids, we adopt the following convention: for $k$ a positive integer, a matroid $M$ is said to be $k$-minor-irreducible, without specifying family, if $M$ is $k$-minor-irreducible in $\mathcal{F}$. To contextualize the reader, we begin by stating the main results of Lemos $[5,6]$ from the viewpoint of minor-irreducibility. Using certain reduction operations, Lemos [5] determines the 3-minor-irreducible matroids with at least 14 elements. In Section 2 we list the 2-minor-irreducible matroids mentioned in this introduction.

Theorem 1.1. (compare with Theorem 1.7 - [5]) Let $M$ be a 3-minorirreducible matroid with at least 14 elements. Then $M$ is isomorphic to:

i) an almost-double-wheel or an almost-double-whirl having rank at least 8;

ii) the graphic matroid of a double-wheel with odd rank exceeding 7 , or to a matroid obtained from a triadic Möbius matroid with even rank exceeding 8 after deleting it's tip;

iii) a (m, n)-triangular matroid, for some non-negative integers $m$ and $n$ with $m+n \geq 2$.

With this, our attention turns to the 2-minor-irreducible matroids that are 3-minor-reducible. Triads and squares, 4-set circuits, plays a fundamental role in the structure of 2-minor-irreducible matroids. The existence of a triad contained in a square is sufficiently restrictive, as shown by Theorem 1.2. A matroid $M$ is said to be semi-binary provided $T^{*} \nsubseteq Q$, for every triad $T^{*}$ and square $Q$ of $M$. Otherwise, $M$ is said non-semi-binary.

Theorem 1.2. (compare with Theorem 1.4-[5]) Let $M$ be a non-semi-binary 2-minor-irreducible matroid with at least 11 elements. Then:

i) $M$ is isomorphic to an almost-double-wheel or an almost-double-whirl; or ii) $M$ is isomorphic to a non-binary ladder or to a relaxed non-binary ladder.

The main result of [6] establish that:

Theorem 1.3. (compare with Theorem 1.2 - [6]) Let $M$ be a 2-minorirreducible matroid with at least 11 elements. If there is an element $e$ in exactly two triads such that $c o(M \backslash e)$ is a triangle-free 3-connected matroid 
then:

i) $M$ is isomorphic to $M(G)$, where $G$ is a ladder or a Möbius ladder graph, if $M$ is semi-binary; or

ii) $M$ is isomorphic a non-binary ladder or to a relaxed non-binary ladder, if $M$ is non-semi-binary.

One of the main result of this paper is a local characterization for semibinary 2-minor-irreducible matroids. It establishes that each element belongs to one of the three configurations of triads and squares described below. Such structures, with the exception of the emerald, are named by Lemos in [5]. They are like 'building-blocks' for semi-binary 2-minor-irreducible matroids.

Sapphire: Let $Q_{1}$ and $Q_{2}$ be distinct squares of $M$ such that $\left|Q_{1} \cap Q_{2}\right|$ $=1$. If $Q_{1} \cap Q_{2}$ belongs to at least 2 triads $T^{*}$ and $T^{*}$ of $M, S=Q_{1} \cup Q_{2}$ is said to be a sapphire with nucleus $Q_{1} \cap Q_{2}$. A sapphire $S$ is called pure when there is another triad $T^{\prime \prime *}$ containing $Q_{1} \cap Q_{2}$ such that $\left|T^{*} \cap T^{* *}\right|=$ $\left|T^{*} \cap T^{\prime \prime *}\right|=\left|T^{* *} \cap T^{\prime \prime *}\right|=1$ and $S$ is closed in both $M$ and $M^{*}$.

Emerald: Let $Q_{1}$ and $Q_{2}$ be distinct squares of $M$ such that $\left|Q_{1} \cap Q_{2}\right|$ $=2$. If there are disjoint triads $T_{1}^{*}$ and $T_{2}^{*}$ of $M$ such that $T_{1}^{*} \cup T_{2}^{*}=Q_{1} \cup Q_{2}$ then $\mathcal{E}=Q_{1} \cup Q_{2}$ is said to be an emerald. If the symmetric difference $Q_{1} \triangle Q_{2}$ is also a square of $M$, we say that $\mathcal{E}$ is pure.

Diamond: Let $Q_{1}, Q_{2}$ and $Q_{3}$ be squares of $M$ such that $\left|Q_{i} \cap Q_{j}\right|=1$, for each 2-subset $\{i, j\}$ of $\{1,2,3\}$, and $Q_{1} \cap Q_{2} \cap Q_{3}=\emptyset$. If $T^{*}=\left(Q_{1} \cap Q_{2}\right) \cup$ $\left(Q_{1} \cap Q_{3}\right) \cup\left(Q_{2} \cap Q_{3}\right)$ is a triad of $M, D=Q_{1} \cup Q_{2} \cup Q_{3}$ is said to be a diamond with nucleus $T^{*}$. If $T^{*}$ does not intersects any other triad of $M$, we say that $D$ is pure.

We are now ready to present one of the main result of this paper. Its proof is in Section 5.

Theorem 1.4. Let $M$ be a semi-binary 2-minor-irreducible matroid with at least 11 elements. For each $e \in E(M)$ there is a triad $T^{*}$ containing e such that $T^{*}$ is contained in a sapphire, or it is contained in an emerald or it is a nucleus of a pure diamond.

Theorems 1.1 to 1.3 determine which are the 2-minor-irreducible matroids that avoid emeralds and pure diamonds, with at least 14 elements. Next result, established in Section 6, deals with 2-minor-irreducible matroids that avoid sapphires and emeralds. We denote by $\mathcal{D}$ the class of 2 -minorirreducible matroids with at least 11 elements that avoids sapphires and 
emeralds. A matroid $M \in \mathcal{D}$ is called diamantic matroid. Theorem 1.5 establishes a bijection between this class of matroids and the class of totally triangular matroids. A 3-connected matroid $M$ is called totally triangular if each of its elements belongs to at least 2 triangles, every pair of triangles intersects in at most 1 element and $M$ has no triads.

Note that if $M$ is totally triangular then $M^{*}$ is a triangle-free 3-connected matroid such that each element belongs to at least 2 triads and each pair of triads of $M^{*}$ intersects in at most 1 element. We denote by $\mathcal{T}$ the class of totally triangular matroids.

Theorem 1.5. There is a bijection $b: \mathcal{D} \longrightarrow \mathcal{T}$ such that if $M$ is a rank $m$ diamantic matroid with $n$ triads then $b(M)$ is a totally triangular matroid with rank $m-n$ and $n$ triangles. Conversely, if $M$ is a rank $m$ totally triangular matroid with $n$ triangles then $b^{-1}(M)$ is a diamantic matroid with $n$ triads and rank $n+m$.

In Section 7, we prove the last result of this paper. A sequence of equivalences on 3-connected matroids covered by pure emeralds.

Theorem 1.6. The following statements are equivalents for a 3-connected matroid $M$, with $|E(M)| \geq 9$, in which each of its elements belongs to a pure emerald:

i) every pair of elements is in a square;

ii) for distinct elements $x$ and $y$ there is a pure emerald containing both;

iii) $M$ is binary;

iv) $M \simeq M\left(K_{3, n}\right)$ where $|E(M)|=3 n$.

This theorem has as a particular case one of the main results of a recent paper due to Oxley, Pfeil, Semple and Whittle [14], in which they establish the equivalence between (i) and (iv). As corollary of Theorem 1.6, we have:

Theorem 1.7. Let $M$ be a non-empty 2-minor-irreducible binary matroid such that each element belongs to an emerald. Then $M \simeq M\left(K_{3, n}\right)$ for some $n \geq 3$.

\section{Known families of 2-minor-irreducible matroids}

In this section we list all known families of 2-minor-irreducible matroids. 


\subsection{Almost-double-wheel and Almost-double-whirl:}

Let $E=\{1,2, \ldots, 2 m, 2 m+1\}$ with $m \geq 5$. Then there are exactly two non-isomorphic triangle-free 3 -connected matroids over $E$ having:

i) $\{1,2,3,4\}$ as a square; and

ii) for every $i \in\{1,2, \ldots, m\},\{2 i-1,2 i, 2 i+1\}$ as a triad; and

iii) for every $i \in\{2,3, \ldots, m-1\},\{2 i-2,2 i-1,2 i+1,2 i+2\}$ as a square.

The subset $I=\{i \in E: i$ is odd $\}$ is a circuit-hyperplane of one of these matroids, which we shall denote by $M$, called an almost-double-wheel. The matroid obtained from $M$ relaxing the circuit-hyperplane $I$ is called an almost-double-whirl. Moreover, we have that $r(M)=m+1, I$ is a Hamiltonian circuit of $M^{*}$ and $P=\{i \in E: i$ is even $\}$ is an independent-hyperplane of $M$. The almost-double-wheel and almost-double-whirl are non-semi-binary 3-minor-irreducible matroids defined and constructed by Lemos in Section 5 of [5]. Follows an auxiliary graph to illustrate these squares and triads:

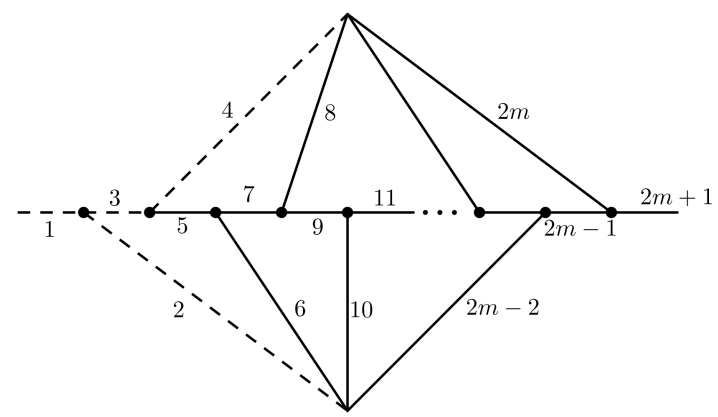

Figure 1: The 3-set of edges incident with vertices of degree 3 illustrate the triads, the edges set of a 4-cycle in this graph represents the squares mentioned in (iii) and the 4 -set of dashed lines is a square containing the triad $\{1,2,3\}$.

\subsection{Double-wheel and Triadic Möbius matroid:}

Let $M\left(W_{n}\right)$ be a $n$-wheel with rank $n \geq 6$. There is just one 3 -connected binary matroid $N$ with ground set $E(N)=E\left(M\left(W_{n}\right)\right) \cup\{e\}$, for a new element $e$, such that $N$ is triangle-free and $N / e=M\left(W_{n}\right)$. When $n$ is even, $N$ is graphic and $D_{n}=N \backslash e$ is called double-wheel with rank $n+1$. When $n$ is odd, the matroid $N$ is called triadic Möbius matroid and the element $e$ called tip of $N$. Mayhew, Royle and Whittle [8] denoted $N$ by $\Upsilon_{n+1}$. Its rank is $n+1$. Double-wheel and triadic Möbius matroid with its tip deleted are both semi-binary 3-minor-irreducible matroids. 


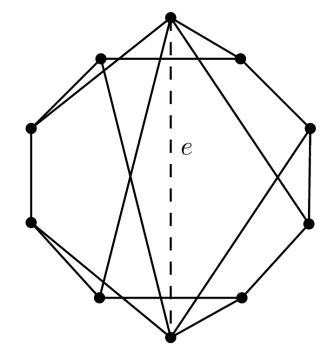

Figure 2: $D_{8}$ denotes the cycle matroid of this graph deleting $e$.

\section{3. (m, n)-triangular matroid:}

A 3-connected matroid $M$ is said to be $(m, n)$-triangular, for non-negative integers $m$ and $n$ such that $m+n \geq 2$, when $M$ is obtained from a matroid $N$ whose ground set is partitioned into $m+n$ triangles, say $T_{1}, \ldots, T_{m}, T_{1}^{\prime}, \ldots T_{n}^{\prime}$, and whose simplification is 3 -connected by:

(i) adding an element $e^{\prime}$ in series with each element $e$ of $N$; and

(ii) for each $i \in\{1, \ldots, m\}$, adding an element $e_{i}$ such that, for every $e \in T_{i}$, $\left\{e_{i}, e, e^{\prime}\right\}$ is a triad of $M$; and

(iii) for each $i \in\{1, \ldots, n\}$, adding elements $e_{i}, f_{i}, g_{i}$ such that $\left\{e_{i}, f_{i}, g_{i}\right\}$, $\left\{e_{i}, a_{i}, a_{i}^{\prime}\right\},\left\{f_{i}, b_{i}, b_{i}^{\prime}\right\}$ and $\left\{g_{i}, c_{i}, c_{i}^{\prime}\right\}$ are triads of $M$, where $T_{i}^{\prime}=\left\{a_{i}, b_{i}, c_{i}\right\}$.
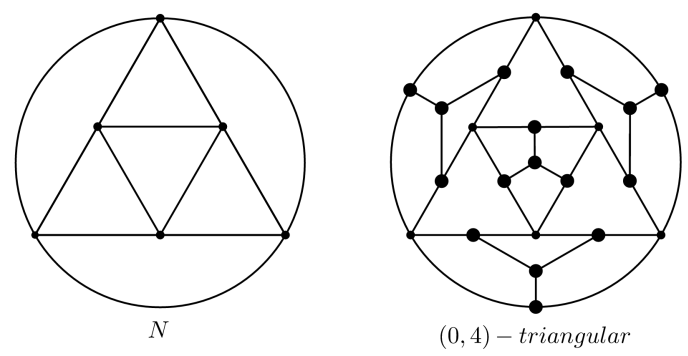

Figure 3: Graph of a $(0,4)$-triangular matroid. Its degree 3 vertices are pointed out.

Rubies: Suppose that there is a triad $T^{*}$ and pairwise disjoint triads $T_{0}^{*}, T_{1}^{*}$ and $T_{2}^{*}$ of a matroid $M$ such that $T^{*} \cap T_{i}^{*}=\left\{e_{i}\right\}, T_{i}^{*}-T^{*}=\left\{f_{i}, g_{i}\right\}$ and $Q_{i}=\left\{e_{i}, g_{i}, e_{i+1}, f_{i+1}\right\}$ is a square of $M$, for every $i \in\{0,1,2\}$ where 
the indices are taken modulus 3 . Then $R=T_{0}^{*} \cup T_{1}^{*} \cup T_{2}^{*}$ is said to be a ruby of $M$ with nucleus $T^{*}$. We say that $R$ is pure provided is closed in both $M$ and $M^{*}$.

The ground set of a $(m, n)$-triangular matroid $M$ is partitioned into $m$ pure sapphires, $S_{i}$ for $i \in\{1, \ldots, m\}$, and $n$ pure rubies, $R_{j}$ for $j \in\{1, \ldots, n\}$.

\subsection{Ladder and Möbius ladder:}

For $n \geq 4$, the ladder $L_{n}$ with $2 n$ vertices is the graph illustrated in Figure 4. We denote by $M\left(L_{n}\right)$ its cycle matroid. If in $L_{n}$ we delete the edges $\left\{T_{1}^{*}, T_{n}^{*}\right\}$ and $\left\{T_{1}^{\prime *}, T_{n}^{\prime *}\right\}$ and we add new edges $\left\{T_{1}^{\prime *}, T_{n}^{*}\right\}$ and $\left\{T_{1}^{*}, T_{n}^{\prime *}\right\}$, then we get the Möbius ladder graph $\mathcal{L}_{n}$ with $2 n$ vertices. We denote by $M\left(\mathcal{L}_{n}\right)$ its cycle matroid.



Figure 4: Ladder $L_{n}$ with $2 n$ vertices. The 3 -set of edges incident in a vertex of degree 3 are triads of cycle matroid associated to $L_{n}$.

\subsection{Non-binary ladder and relaxed non-binary ladder:}

For $n \geq 4$, let $G_{n}$ be the auxiliary graph displayed in Figure 5 . Set $D=\left\{a_{1}, a_{2}, \ldots, a_{n}, b_{1}, b_{2}, \ldots, b_{n}\right\}$. Then the relaxed non-binary ladder $R_{n}$ of rank $2 n$ is a matroid over $E\left(G_{n}\right)$ such that $\mathcal{C}\left(R_{n}\right)=\mathcal{C} \cup \mathcal{D}$, where i) $C \in \mathcal{C}$ if and only if $C$ is a circuit of the cycle matroid associated with $G_{n}$ and $C \neq D \cup\left\{c_{0}, c_{n}\right\}$; and

ii) $C \in \mathcal{D}$ if and only if $C=E(T)$, where $T$ is a tree of $G_{n}$ such that: each leaf vertex of $T$ is incident in $G_{n}$ with $c_{0}$ or $c_{n}$, and every vertex incident with $c_{0}$ or $c_{n}$ in $G_{n}$ is a vertex of $T$. 


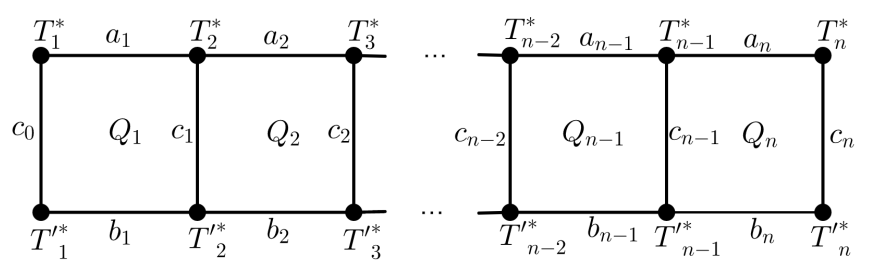

Figure 5: Auxiliary graph $G_{n}$.

The $2 n$-set $D$ is a basis of $R_{n}$. There is a matroid $P_{n}$ over $E\left(G_{n}\right)$ such that

$$
\mathcal{C}\left(P_{n}\right)=\left[\mathcal{C}\left(R_{n}\right)-\left\{D \cup c_{i} \mid 0 \leq i \leq n\right\}\right] \cup\{D\}
$$

and $R_{n}$ is obtained from $P_{n}$ by relaxing the circuit-hyperplane $D$. We say that $P_{n}$ is the non-binary ladder of rank $2 n$. The non-binary-ladder and relaxed non-binary-ladder are both non-semi-binary 2-minor-irreducible matroids constructed by Lemos in Section 2 of [6].

\subsection{Diamantic matroids:}

A 3-connected matroid $M$ is called a diamantic matroid if each of its elements belongs to a nucleus of a pure diamond and $M$ has no emeralds. There is a bijection $b$ from the class of diamantic matroids to the class of totally triangular matroids such that for each rank $m$ diamantic matroid $M$ with $n$ triads, $b(M)$ is a totally triangular matroid with rank $m-n$ and $n$ triangles. Since $P_{7}$, displayed in Figure 8 , is the totally triangular matroid with fewest triangles, we have $b^{-1}\left(P_{7}\right)$ the smallest diamantic matroid. Follows a graph representation of a diamantic matroid and its totally triangular matroid associated.


Figure 6: The cycle matroid of $G$ is a diamantic matroid such that its totally triangular matroid associated is the cycle matroid of $b(G)$. Degree 3 vertices of $G$ are pointed out. 
2.7. The cycle matroid of bipartite graph $M\left(K_{3, n}\right)$, for $n \geq 3$ :

In this context of minor-irreducibility, this is the family of binary 2-minorirreducible matroids such that each element belongs to an emerald. Note that $M\left(K_{3,3}\right)$ is a 3 -minor-irreducible matroid.

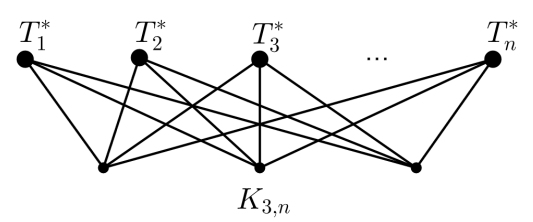

Figure 7: The bipartite graph $K_{3, n}$.

\section{Preliminary results}

We use the terminologies and notation set in [12]. If $M$ denotes a matroid, its connectivity function is

$$
\lambda: 2^{E(M)} \longrightarrow \mathbb{N}
$$

such that for $X \subseteq E(M)$

$$
\begin{aligned}
\lambda(X) & =r(X)+r(E(M)-X)-r(M) \\
& =r(X)+r^{*}(X)-|X|
\end{aligned}
$$

where $r$ is the rank function of $M$ and $r^{*}$ the rank function of $M^{*}$. A subset $X \subseteq E(M)$ is a $k$-separating set, for $k \geq 1$, if

$$
\lambda(X) \leq k-1 \leq \min \{|X|,|E(M)-X|\}-1
$$

If $X$ is a $k$-separating set, the partition $\{X, E(M)-X\}$ is said a $k$ separation. A $k$-separation $\{X, E(M)-X\}$ is exact if $\lambda(X)=k-1$. A matroid $M$ is said $n$-connected if it has no $k$-separation for $k<n$. If $M$ is a $n$-connected matroid, a $n$-separation $\{X, E(M)-X\}$ is called trivial if $\min \{|X|,|E(M)-X|\}=n$. 


\subsection{Knowns results on 3-connected matroids.}

We start with some key results on 3-connected matroids. From Lemos [4], we use the following result:

Theorem 3.1. Let $M$ be a 3-connected matroid and $C$ a circuit of $M$ such that $M \backslash e$ is not 3-connected for all $e \in C$. Then there are at least two triads of $M$ intersecting $C$.

Most times we only need a weaker version of the above result, due to Oxley [9]:

Theorem 3.2. Each circuit of a minimally 3-connected matroid meets at least two triads.

The main result of Bixby [2]:

Theorem 3.3. (Bixby's Theorem) If $M$ is 3-connected and e $\in E(M)$, then: i) Every 2-separation for $M \backslash e$ is trivial and so co $(M \backslash e)$ is 3-connected; or ii) Every 2-separation for $M /$ e is trivial and so si $(M / e)$ is 3-connected.

The Tuttes's Triangle Lemma:

Lemma 3.4. (Tuttes's Triangle Lemma) Let $M$ be a 3-connected matroid having at least 4 elements and suppose that $\{e, f, g\}$ is a triangle of $M$ such that neither $M \backslash e$ nor $M \backslash f$ is 3-connected. Then $M$ has a triad that contains $e$ and exactly one of $f$ and $g$.

From Oxley [10], we use the following result:

Lemma 3.5. Suppose that $e$ and $f$ are distinct elements of a $n$-connected matroid $M$ with $|E(M)| \geq 2(n-1), n \geq 2$. Assume that $M / e \backslash f$ is $n$ connected but $M \backslash f$ is not. Then $M$ has a cocircuit with length $n$ containing $e$ and $f$.

\subsection{Reduction operations on triangle-free 3-connected matroids.}

Consider the following reduction operations on $\mathcal{F}$, operations that when applied to the elements of a matroid $M \in \mathcal{F}$ produce a minor $N<M$ and $N \in \mathcal{F}$. The first two reduction operations are: 
- First reduction: A triangle-free 3-connected matroid $M$ is called 1-reducible if there is an element $e$ such that $M \backslash e$ is a triangle-free 3-connected matroid. Otherwise, $M$ is said to be 1-irreducible.

- Second reduction: A triangle-free 3-connected matroid $M$ is called 2-reducible if there is $e \in E(M)$ such that $M / e$ is a triangle-free 3connected matroid. If $M / e$ is not a triangle-free 3-connected matroid for every element $e \in E(M), M$ is said 2-irreducible.

A triangle-free 3-connected matroid $M$ is called 12-irreducible if it is both 1-irreducible and 2-irreducible. Therefore, $M$ is 12-irreducible if and only if $M$ is 1-minor-irreducible in $\mathcal{F}$. Classifying 1-minor-irreducible matroids in $\mathcal{F}$ is an unviable task. For this reason, we shall consider another reduction operation:

- Third reduction: A triangle-free 3-connected matroid $M$ is called 3-reducible when there are squares $Q_{1}$ and $Q_{2}$ intersecting in a single element, say $f$, belonging to a unique triad such that $c o(M \backslash f)$ is triangle-free 3-connected. Otherwise, $M$ is said 3-irreducible.

A triangle-free 3-connected matroid $M$ is called 123-irreducible if it is $i$ irreducible for every $i \in\{1,2,3\}$. Despite the similarity between $i$-irreducible and $i$-minor-irreducible notations, for $1 \leq i \leq 3$, we decided to keep the notation used by Lemos [5]. We hope it does not cause confusion for the reader. The following lemmas are both in Section 2 of Lemos [5].

Lemma 3.6. Let $M$ be an 1-irreducible matroid with $|E(M)| \geq 7$. If $Q_{1}$ and $Q_{2}$ are different squares of $M$, then $\left|Q_{1} \cap Q_{2}\right| \leq 2$.

Lemma 3.7. Suppose that $M$ is a semi-binary 2-irreducible matroid. Then each coline of $M$ has at most 3 elements.

\subsection{Fullclosure operator and sequential separation.}

The terminologies for fullclosure operator and sequential separations were introduced by Oxley, Semple and Whittlel [13]. Let $M$ be a matroid. We define the fullclosure operator as the function $f c l_{M}: 2^{E(M)} \longrightarrow 2^{E(M)}$ such that

$$
f c l_{M}(X)=\min \left\{Z \subseteq E(M) \mid X \subseteq Z=c l(Z)=c l^{*}(Z)\right\}
$$


where $c l$ and $c l^{*}$ denotes, respectively, the closure and coclosure operator of $M$. Note that $f c l_{M}(X)=f_{c l}(X)$. We denote by $f c l(X)$ when it does not cause confusion.

One way of obtaining the fullclosure of a subset $X \subseteq E(M)$ is to take alternately closure and coclosure and so on until neither the closure nor the coclosure operator adds new elements. The elements of $f c l(X)-X$ can be ordered

$$
f c l(X)-X=\left\{x_{1}, \ldots, x_{n}\right\}
$$

such that $x_{i} \in \operatorname{cl}\left(X \cup\left\{x_{1}, \ldots, x_{i-1}\right\}\right)$ or $x_{i} \in c l^{*}\left(X \cup\left\{x_{1}, \ldots, x_{i-1}\right\}\right)$. The following result hold for $k$-separating sets with $k \geq 1$, but our only interest is in the case $k=2$ and 3 :

Lemma 3.8. (Lemma 3.1 - [13]) Let $\{X, Y\}$ be an exact $k$-separation for a matroid $M$.

i) For $e \in Y$, the partition $\{X \cup e, Y-e\}$ is a k-separation if and only if $e \in c l(X)$ or $e \in c l^{*}(X)$;

ii) For $e \in Y$, the partition $\{X \cup e, Y-e\}$ is an exact $k$-separation if and only if

$$
e \in[c l(X) \cap c l(Y-e)] \triangle\left[c l^{*}(X) \cap c l^{*}(Y-e)\right] ;
$$

iii) The elements of $f c l(X)-X$ can be ordered $\left\{x_{1}, \ldots, x_{n}\right\}$ such that $X \cup$ $\left\{x_{1}, \ldots, x_{i}\right\}$ is k-separating for all $i \in\{1, \ldots, n\}$.

A $k$-separation $\{X, Y\}$ for a matroid $M$ is said sequential provided $f c l(X)=E(M)$ or $f c l(Y)=E(M)$. Otherwise, $\{X, Y\}$ is said nonsequential.

Example 3.9. A trivial 2-separation $\{X, Y\}$ for a connected matroid $M$ is also sequential.

From Lemos [5], we have:

Lemma 3.10. (Lemma 3.1 - Lemos [5]) Suppose that $M$ is a triangle-free 3-connected matroid with at least 5 elements. Given $e \in E(M)$, every 2separation for $M / e$ is non-sequential.

Lemma 3.11. (Lemma 3.2 - Lemos [5]) Suppose that $M$ is a triangle-free 3-connected matroid and $e \in E(M)$. If $\{X, Y\}$ is a non-trivial 2-separation for $M \backslash e$ then $\{X, Y\}$ is non-sequential or e belongs to a coline with at least 4 elements. Moreover, when $M$ is also semi-binary and 2-irreducible, $\{X, Y\}$ is non-sequential. 


\subsection{Forced Sets.}

This subsection contains some results set out in Section 4 of [5]. They are used to establish the auxiliary results in the next section. Let $M$ be a matroid with ground set $E(M)$. A subset $F \subseteq E(M)$ is forced provided, for every $e \in E(M)-F$ and 2-separation $\{X, Y\}$ for any $N \in\{M \backslash e, M / e\}$, there is $Z \in\{X, Y\}$ such that $F \subseteq f c l_{N}(Z)$.

Forced sets are not separated by 2 -separations on matroids resulting from contraction or deletion of elements outside $F$.

Example 3.12. If for every $W \subseteq F, F \subseteq f c l_{M}(W)$ or $F \subseteq f c l_{M}(F-W)$ then $F$ is a forced set of $M$.

Lemma 3.13. If $F$ is a forced set of $M$ and $e$ is spanned by $F$ in $M$ or $M^{*}$ then $F \cup e$ is a forced set in $M$.

Lemma 3.14. Suppose that $M$ is a triangle-free 3-connected matroid with at least 5 elements and $F$ is a forced set of $M$. If $e \in c l^{*}(F)-F$ then $M / e$ is 3-connected. Moreover, when $M$ is 2-irreducible there is a square $Q$ of $M$ that contains e.

Lemma 3.15. Suppose that $F$ is a forced set of a triangle-free 3-connected matroid $M$. If e $\in \mathrm{cl}(F)-F$ then:

i) every 2-separation for $M \backslash e$ is trivial and so co $(M \backslash e)$ is 3-connected; or

ii) e belongs to a coline with at least 4 elements.

Moreover:

iii) when (ii) occurs, $e$ is spanned by $F$ in both $M$ and $M^{*}$;

iv) when $M$ is semi-binary and 2-irreducible, (i) happens.

If $M$ is 1-irreducible, $F$ a forced set of $M$ and $e \in c l(F)-F$ then $e$ belongs to a triad of $M$.

Lemma 3.16. Let $M$ be a triangle-free 3-connected matroid and $F$ a 3separating forced set of $M$. Suppose that $M \mid F$ is coloopless and $|E(M)-F| \geq$ 5. If $C^{*}$ is a cocircuit of $M$ such that $\left|C^{*}-F\right|=1$ and each element of $C^{*} \cap F$ belongs to a triad contained in $F$ then $M$ is (1 or 2 or 3)-reducible. 


\section{Auxiliary lemmas on intersection of squares}

The importance of squares and triads for triangle-free 3-connected matroids has already been mentioned. In this section, we have established four auxiliary lemmas dealing with certain configurations of squares and triads. These results are used in the next section, where we prove Theorem 1.4. Each subsection is dedicated to one of these configurations.

\subsection{A pair of squares having just one element in common.}

Lemma 4.1. Let $M$ be a minimally 3-connected matroid with $Q_{1}$ and $Q_{2}$ squares of $M$ such that $Q_{1} \cap Q_{2}=\{f\}$. Suppose that $f$ belongs to a unique triad $T^{*}=\{e, f, g\}$. We can assume that $e \in Q_{1}$ and $g \in Q_{2}$. If $M / e$ is 3-connected and if there is a triad $T^{*}$ containing $Q_{2}-T^{*}$ then co $(M \backslash f)=$ $M \backslash f / e$ is 3-connected.

Proof. Suppose that co $(M \backslash f)=M \backslash f / e$ is not 3-connected. Tutte's Triangle Lemma 3.4 implies that $M / e \backslash x$ is 3-connected for each $x \in Q_{1}-T^{*}$, otherwise there is a triad of $M / e$ containing $\{f, x\}$. Using Bixby's Theorem 3.3, we have that there is a non-trivial (exact) 2-separation for $M \backslash f$, say $\{X, Y\}$. Suppose that $\{X, Y\}$ is sequential. Lemma 3.8 implies that we can put an order on $X$ or $Y$, say $Y=\left\{y_{1}, \ldots, y_{n-2}, y_{n-1}, y_{n}\right\}$, with $n \geq 3$, such that $\left\{y_{n-2}, y_{n-1}, y_{n}\right\}$ and $\left\{y_{n-1}, y_{n}\right\}$ are both 2-sparating set for $M \backslash f$. Then $\left\{y_{n-1}, y_{n}\right\}$ is in a series class of $M \backslash f,\left\{y_{n-1}, y_{n}\right\}=\{e, g\}$, and so $\left\{y_{n-2}, e, g,\right\}$ is a triad of $M$. Hence $y_{n-2} \in Q_{1} \cap Q_{2}$, because of orthogonality; a contradiction. Therefore $\{X, Y\}$ is non-sequential.

Denote by $T_{x}^{*}$ and $T_{y}^{*}$ the triads of $M$ that contains $\{e, x\}$ and $\{e, y\}$, respectively, where $\{x, y\}=Q_{1}-T^{*}$. We can suppose that $\left|X \cap T_{x}^{*}\right| \geq$ 2. If $y \in X$ then $f$ belongs to $c l(X)$, contradicting 3-connectivity of $M$. So $\left|T_{y}^{*} \cap Y\right| \geq 2$, we have that $g \in X$ and $\left|T^{*} \cap Y\right| \geq 2$. Therefore $T^{*} \cup\{e, g\} \subseteq c l^{*}(Y), Y \cup T^{*} \cup\{e, g\}$ is a 2-separating set for $M \backslash f$ and $f \in c l\left(Y \cup T^{*} \cup\{e, g\}\right)$; a contradiction. Thus $c o(M \backslash f)=M \backslash f / e$ is 3connected.

4.2. A pair of squares having two elements in common. 
First, note that if $T^{*}$ is a triad of a semi-binary matroid $M$ that intersects $Q_{1} \cup Q_{2}$, where $Q_{1}$ and $Q_{2}$ are squares of $M$ such that $\left|Q_{1} \cap Q_{2}\right|=2$, then: i) $T^{*} \cap F \in\left\{Q_{1}-Q_{2}, Q_{2}-Q_{1}, Q_{1} \cap Q_{2}\right\}$; or

ii) $T^{*} \subseteq F$, and so $\left|T^{*} \cap\left(Q_{1}-Q_{2}\right)\right|=\left|T^{*} \cap\left(Q_{2}-Q_{1}\right)\right|=\left|T^{*} \cap\left(Q_{1} \cap Q_{2}\right)\right|$ $=1$.

Lemma 4.2. Let $M$ be a semi-binary triangle-free 3-connected matroid with at least 9 elements. If $Q_{1}$ and $Q_{2}$ are squares of $M$ such that $\left|Q_{1} \cap Q_{2}\right|=2$ and $F=Q_{1} \cup Q_{2}$ contains at least two triads of $M$, then $F$ is a 3-separating forced set of $M$ with $r(F)=r^{*}(F)=4$.

Proof. Let $T^{*}$ and $T^{*}$ be distinct triads contained in $F=Q_{1} \cup Q_{2}$. We have $r(F) \leq 4$, because of squares $Q_{1}$ and $Q_{2}$, and $r^{*}(F) \leq 4$, because of triads $T^{*}$ and $T^{*}$. Hence $\lambda(F) \leq 2$ and $|E(M)-F| \geq 3$. The 3-connectivity of $M$ implies that $\lambda(F)=2$ and so $F$ is a 3-separating set with $r(F)=r^{*}(F)=4$. If $\left|T^{*} \cap T^{*}\right|=2$ then $L^{*}=T^{*} \cup T^{*}$ is contained in a coline and so $M$ has a square containing a triad; a contradiction. If $\left|T^{*} \cap T^{*}\right|=1$ then $F$ is a forced set of $M$, because for each subset $W \subseteq F$ we have $F \subseteq f c l(W)$ or $F \subseteq f c l(F-W)$.

Finally, we can assume that $T^{*} \cap T^{*}=\emptyset$ and so $F$ is an emerald. Suppose that $F$ is not a forced set of $M$. There are $e \in E(M)-F$, $N \in\{M \backslash e, M / e\}$ and $\{X, Y\}$ 2-separation for $N$ such that $F \nsubseteq f c l_{N}(X)$ and $F \nsubseteq f c l_{N}(Y)$. We have $|X \cap F|=|Y \cap F|=3$ and $f_{c l}(X \cap F) \cap F=$ $X \cap F$. The same holds for $Y$. Then $\{X \cap F, Y \cap F\}=\left\{T^{*}, T^{*}\right\}$ and $\{X \cap F, Y \cap F\}$ is a 2-separation for $N \mid F$. As $\left\{T^{*}, T^{\prime *}\right\}$ is not a 2-separation for $M \mid F$, it follows that $N=M / e$. Denoting by $M^{\prime}=M \mid(F \cup\{e\})$, we have $N \mid F=M^{\prime} / e$. Then $\lambda_{M^{\prime} / e}\left(T_{1}^{*}\right)=\lambda_{N \mid F}\left(T_{1}^{*}\right)=1$, with $r\left(M^{\prime}\right)=5$ and $\min \left\{r_{M^{\prime}}\left(T_{1}^{*} \cup e\right), r_{M^{\prime}}\left(T_{2}^{*} \cup e\right)\right\}=3$. Therefore $M$ has a square containing a triad; a contradiction.

Lemma 4.3. Let $M$ be a semi-binary 123-irreducible matroid with at least 11 elements. If $T^{*}$ and $T^{*}$ are triads of $M$ such that $F=T^{*} \cup T^{*}=Q_{1} \cup Q_{2}$ is an emerald then $M \backslash T^{*}$ is 3-connected and so $M$ is 3-minor-reducible.

Proof. Denote by $T^{*}=\left\{e_{0}, e_{1}, e_{2}\right\}$ and $T^{*}=\left\{e_{0}^{\prime}, e_{1}^{\prime}, e_{2}^{\prime}\right\}$ such that $Q_{0}=$ $\left\{e_{0}, e_{1}, e_{0}^{\prime}, e_{1}^{\prime}\right\}$ and $Q_{1}=\left\{e_{1}, e_{2}, e_{1}^{\prime}, e_{2}^{\prime}\right\}$ are squares of $M$. Suppose $M \backslash T^{*}$ is not 3-connected. Let $\{X, Y\}$ be a $k$-separation for $M \backslash T^{*}$, with $k=1$ or $k=2$. We can suppose $\left|X \cap T^{*}\right| \geq 2$. If $|Y|=1$ then $k=1$ and $Y$ is a coloop of $M \backslash T^{* *}$ and $T^{*} \cup Y$ is a coline, contradicting Lemma 3.7. Thus $|Y| \geq 2$. 
Denote by $M^{\prime}=M \backslash\left(T^{*}-e_{0}^{\prime}\right)$. We have $k=\lambda_{M \backslash T^{* *}}(X)+1 \geq$ $\lambda_{M^{\prime}}\left(X \cup T^{*} \cup e_{0}^{\prime}\right)+1$. Then $\left\{X \cup T^{*}, Y-T^{*}\right\}$ is a $k$-separation for $M \backslash T^{\prime *}$ or $\left|Y-T^{*}\right|<k \leq 2$. If $\left\{X \cup T^{*}, Y-T^{*}\right\}$ is a $k$-separation for $M \backslash T^{* *}$, as $r_{M}\left(T^{*} \cup e_{0}^{\prime}\right)=4$, we have $F \subseteq f c l_{M}\left(X \cup T^{*} \cup e_{0}^{\prime}\right)$ and so $k>$ $\lambda_{M}\left(X \cup T^{*} \cup T^{*}\right)$. Hence $\left\{X \cup T^{*} \cup T^{* *}, Y-T^{*}\right\}$ is a $k$-separation for $M$, which is a contradiction. Therefore $\left|Y-T^{*}\right|=1$ and $|Y|=2$. There is a cocircuit $C^{*}$ of $M$ such that $Y=C^{*}-T^{*}$. Since $F$ is a 3 -separating forced set of $M$ and $\left|C^{*}-F\right|=1$, Lemma 3.16 implies that $M$ is $i$-reducible for some $i \in\{1,2,3\}$; a contradiction.

Lemma 4.4. Let $M$ be a semi-binary 123-irreducible matroid with $|E(M)| \geq$ 11. Let $Q_{1}$ and $Q_{2}$ be squares of $M$ such that $\left|Q_{1} \cap Q_{2}\right|=2$. Then 1) $Q_{1} \cup Q_{2}$ is an emerald; or

2) there are just 3 triads $T_{i}^{*}, i \in\{1,2,3\}$, intersecting $F=Q_{1} \cup Q_{2}$ such that

$$
\left\{T_{1}^{*} \cap F, T_{2}^{*} \cap F, T_{3}^{*} \cap F\right\}=\left\{Q_{1}-Q_{2}, Q_{2}-Q_{1}, Q_{1} \cap Q_{2}\right\},
$$

$Q_{3}=Q_{1} \triangle Q_{2}$ is a square of $M$ and, for each $i \in\{1,2,3\}$, we have:

2.i) $T_{i}^{*} \cup T_{j}^{*}$ is a pure emerald $\mathcal{E}$, for some $j \in\{1,2,3\}-\{i\}$, such that $\mathcal{E} \cap F \in\left\{Q_{1}, Q_{2}, Q_{3}\right\}$; or

2.ii) $T_{i}^{*}$ is contained in an emerald $\mathcal{E}_{i}$ such that $\mathcal{E}_{i} \cap F=T_{i}^{*} \cap F$; or

2.iii) $T_{i}^{*}$ is a nucleus of a pure diamond $D$ such that $D \cap F \in\left\{Q_{1}, Q_{2}, Q_{3}\right\}$; or

2.iv) $T_{i}^{*}-F$ is a nucleus of a sapphire $S_{i}$ such that $S_{i} \cap F=T_{i}^{*} \cap F$.

Proof. Here, $M$ denotes a semi-binary 123-irreducible matroid with at least 11 elements. Assume that $Q_{1}$ and $Q_{2}$ are squares of $M$ such that $\left|Q_{1} \cap Q_{2}\right|=$ 2 and $Q_{1} \cup Q_{2}$ is not an emerald. Denote by $F=Q_{1} \cup Q_{2}$. Using an argument similar to that found in Lemos [5], Section 8, we have:

Sub-lemma 4.4.1. F contains at most one triad of $M$ and there are disjunct triads $T_{i}^{*}$, for each $i \in\{1,2,3\}$, such that

$$
\left\{T_{1}^{*} \cap F, T_{2}^{*} \cap F, T_{3}^{*} \cap F\right\}=\left\{Q_{1}-Q_{2}, Q_{2}-Q_{1}, Q_{1} \cap Q_{2}\right\}
$$

and $Q_{3}=Q_{1} \triangle Q_{2}$ is also a square of $M$. Moreover, for $i \in\{1,2,3\}$, $M /\left(T_{i}^{*}-F\right)$ is 3-connected. 
Denote by $T_{i}^{*} \cap F=\left\{f_{i}, g_{i}\right\}$ and $\left\{e_{i}\right\}=T_{i}^{*}-F$, for $i \in\{1,2,3\}$.

Sub-lemma 4.4.2. For each $i \in\{1,2,3\}$ there are squares $Q_{i}^{\prime}$ containing $e_{i}$ such that:

i) $\left|Q_{i}^{\prime} \cap F\right|=1$; or

ii) for some $j \in\{1,2,3\}-\{i\}, T_{i}^{*} \cup T_{j}^{*}=\mathcal{E}$ is an emerald such that $Q_{i}^{\prime} \subseteq \mathcal{E}$ and $\mathcal{E} \cap F \in\left\{Q_{1}, Q_{2}, Q_{3}\right\}$.

Moreover, if $T_{i}^{*} \cup T_{j}^{*}$ is not an emerald for each 2-subset $\{i, j\}$ contained in $\{1,2,3\}$, then $\left|Q_{k}^{\prime} \cap Q_{l}^{\prime}\right| \leq 1$ for $k \neq l$.

Proof. Since $M$ is 2-irreducible, there is a square $Q_{i}^{\prime}$ containing $e_{i}$. For $i=1$, assume $\left|Q_{1}^{\prime} \cap F\right|>1$. As $\left|Q_{1}^{\prime} \cap T_{1}^{*}\right|=2$, we have: $\left|Q_{1}^{\prime} \cap T_{2}^{*}\right|=2$ and $T_{1}^{*} \cup T_{2}^{*}=Q_{1}^{\prime} \cup Q_{3}$ is an emerald, or $\left|Q_{1}^{\prime} \cap T_{3}^{*}\right|=2$ and $T_{1}^{*} \cup T_{3}^{*}=Q_{1}^{\prime} \cup Q_{1}$ is an emerald.

Suppose that (ii) does not occur. Assume, by contradiction, that $\left|Q_{1}^{\prime} \cap Q_{2}^{\prime}\right|$ $=2$. We have that $\left|Q_{i}^{\prime} \cap F\right|=1$. Then $Q_{1}^{\prime} \cap Q_{2}^{\prime}=Q_{i}^{\prime}-T_{i}^{*}$, for $i=1$ and $i=2$. There is a triad $T^{*}$ such that $T^{*} \cap\left(Q_{1}^{\prime} \cup Q_{2}^{\prime}\right)=Q_{1}^{\prime} \cap Q_{2}^{\prime}$. Then $Q_{1}^{\prime} \triangle Q_{2}^{\prime}$ is a square of $M$ such that $\left|\left(Q_{1}^{\prime} \triangle Q_{2}^{\prime}\right) \cap Q_{3}\right|=2$ and so $T_{1}^{*}$ and $T_{2}^{*}$ are both contained in $\left(Q_{1}^{\prime} \triangle Q_{2}^{\prime}\right) \cup Q_{3}$; a contradiction since $T_{1}^{*} \cup T_{2}^{*}$ is not an emerald.

Sub-lemma 4.4.3. For each $i \in\{1,2,3\}$, if $\left|Q_{i}^{\prime} \cap F\right|=1$ then $T_{i}^{*}$ is the unique triad of $M$ that contains $Q_{i} \cap Q_{i}^{\prime}$.

Proof. Suppose, for $i=1$, that $T^{*}$ is another triad, different from $T_{1}^{*}$, such that $Q_{1} \cap Q_{1}^{\prime} \subseteq T^{*}$. Since $\left|T^{*} \cap T_{1}^{*}\right| \leq 1$, because of Lemma 3.7, $T^{*}$ is contained in $F$ and $\left|Q_{1}^{\prime} \cap F\right|>1$; a contradiction.

Suppose $\left|Q_{i}^{\prime} \cap F\right|=1$, for some $i \in\{1,2,3\}$. Denote by $\left\{f_{i}\right\}=Q_{i} \cap Q_{i}^{\prime}$ and $\left\{g_{i}\right\}=T_{i}^{*}-\left\{e_{i}, f_{i}\right\}$. Lemma 4.1 implies that $c o\left(M \backslash f_{i}\right)=M \backslash f_{i} / e_{i}$ is 3 connected. As $M$ is 3 -irreducible, there is a square $Q_{i}^{\prime \prime}$ containing $e_{i}$ avoiding $f_{i}$. By orthogonality with $T_{i}^{*}$, we have $g_{i} \in Q_{i}^{\prime \prime}$. We have $\left|Q_{i}^{\prime \prime} \cap F\right|=1$, otherwise $T_{i}^{*} \cup T_{j}^{*}$ is an emerald for some $j \in\{1,2,3\}-\{j\}$. Previous sub-lemma implies that $T_{i}^{*}$ is the unique triad containing $g_{i}$.

Suppose $\left|Q_{i}^{\prime} \cap Q_{i}^{\prime \prime}\right|=1$. If $T_{i}^{*}$ is the unique triad containing $e_{i}$, then $Q_{i} \cup$ $Q_{i}^{\prime} \cup Q_{i}^{\prime \prime}$ is a pure diamond with nucleus $T_{i}^{*}$ intersecting $F$ in $Q_{i}$. Otherwise, $Q_{i}^{\prime} \cup Q_{i}^{\prime \prime}$ is a sapphire with nucleus $e_{i}$ that intersects $F$ in $T_{i}^{*}-\left\{e_{i}\right\}$. 
If $\left|Q_{i}^{\prime} \cap Q_{i}^{\prime \prime}\right|=2$, then $Q_{i}^{\prime} \cup Q_{i}^{\prime \prime}$ is an emerald intersecting $F$ in $T_{i}^{*}-\left\{e_{i}\right\}$. Suppose false. Sub-lemma 4.2 implies that $T_{i}^{*}$ is the unique triad contained in $Q_{i}^{\prime} \cup Q_{i}^{\prime \prime}$. Theorem 3.1 implies that there is a triad $T^{*}$ intersecting $Q_{i}^{\prime} \cup Q_{i}^{\prime \prime}$ in addition to $T_{i}^{*}$. As $T_{i}^{*}$ is the unique triad intersecting $\left\{f_{i}, g_{i}\right\}, T_{i}^{*} \cap$ $\left(Q_{i}^{\prime} \cup Q_{i}^{\prime \prime}\right)=Q_{i}^{\prime} \cap Q_{i}^{\prime \prime}$ and $Q_{i}^{\prime} \triangle Q_{i}^{\prime \prime}$ is a square of $M$ intersecting only one triad; a contradiction.

\subsection{Pure diamonds.}

Lemma 4.5. Let $M$ be a semi-binary 123-irreducible matroid with $|E(M)| \geq$ 11. Let $D=Q_{1} \cup Q_{2} \cup Q_{3}$ be a pure diamond with nucleus $T^{*}$. Denote by $T_{i}^{*}$, for $i \in\{1,2,3\}$, triads such that $T_{i}^{*} \cap D=Q_{i}-\left(Q_{j} \cup Q_{k}\right)$ with $\{i, j, k\}=\{1,2,3\}$. Then for each $i \in\{1,2,3\}$ :

i) $T_{i}^{*} \cup T^{*}$ is an emerald; or

ii) $T_{i}^{*}$ is contained in an emerald $\mathcal{E}_{i}$ such that $\mathcal{E}_{i} \cap D=T_{i}^{*} \cap D$; or

iii) $T_{i}^{*}$ is a nucleus of a pure diamond $D_{i}$; or

iv) $T_{i}^{*}-D$ is a nucleus of a sapphire $S_{i}$ such that $S_{i} \cap D=T_{i}^{*} \cap D$.

Moreover, $M \backslash T^{*}$ is 3-connected and $M$ is 3-minor-reducible.

Proof. By dual form of Tutte's Triangle Lemma 3.4, there is $f_{i} \in T_{i}^{*} \cap Q_{i}$ such that $M / f_{i}$ is 3 -connected, for every $i \in\{1,2,3\}$. Bixby's Theorem 3.3 implies that $M / f_{i} / e_{i}$ is 3-connected, where $\left\{e_{i}\right\}=T_{i}^{*}-Q_{i}$. Therefore, $M / e_{i}$ is 3 -connected.

As $M$ is 2-irreducible, there is a square $Q_{1}^{\prime}$ containing $e_{i}$. If $\left|Q_{i} \cap Q_{i}^{\prime}\right|=2$, $Q_{1} \cup Q_{1}^{\prime}=T^{*} \cup T^{* *}$ is an emerald, because of Lemma 4.4. Suppose $\left|Q_{i} \cap Q_{i}^{\prime}\right|=$ 1 and take $\left\{g_{i}\right\}=Q_{i} \cap Q_{i}^{\prime}$. By Lemma 4.1, $M \backslash g_{i} / e_{i}$ is 3-connected and then there is a square $Q_{i}^{\prime \prime}$ containing $e_{i}$ avoiding $g_{i}$. We can suppose $\left|Q_{i} \cap Q_{i}^{\prime \prime}\right|=1$, otherwise $T^{*} \cup T^{\prime *}$ is an emerald. If $\left|Q_{i}^{\prime} \cap Q_{i}^{\prime \prime}\right|=2$, Lemma 4.1 implies that $Q_{i}^{\prime} \cup Q_{i}^{\prime \prime}$ is an emerald intersecting $D$ in $T_{i}^{*} \cap D$. If $\left|Q_{i}^{\prime} \cap Q_{i}^{\prime \prime}\right|=1$ and $T_{i}^{*}$ is the unique triad containing $e_{i}$, then $Q_{i} \cup Q_{i}^{\prime} \cup Q_{i}^{\prime \prime}$ is a pure diamond with nucleus $T_{i}^{*}$. Otherwise, $Q_{i}^{\prime} \cap Q_{i}^{\prime \prime}$ is a sapphire with nucleus $e_{i}$. The same argument used in Lemma 6.3 of [5] shows that $M \backslash T^{*}$ is 3-connected.

\section{Proof of Theorem 1.4: local characterization for 2-minor- irreducible matroids}

This section contains a sequence of results that constitute the proof of Theorem 1.4. Here, $M$ denotes a semi-binary 123-irreducible matroid with 
at least 11 elements. We denote by $\mathcal{S}$ the union of sapphires, pure diamonds and emeralds of $M$. Our goal is to show that $\mathcal{S}=E(M)$.

Lemma 5.1. Let $Q$ be a square of $M$ and $T^{*}$ a triad such that $T^{*} \cap Q \neq \emptyset$. If $e \in Q-T^{*}$, then:

i) $M / e$ is not 3-connected. In this case there is a triad containing e; or

ii) $M / e$ is 3-connected and there is a square $Q^{\prime}$ such that $T^{*} \subseteq Q \cup Q^{\prime}$ and $\left|Q \cap Q^{\prime}\right|=1$; or

iii) $M / e$ is 3-connected and there is a square $Q^{\prime}$ such that $T^{*} \subseteq Q \cup Q^{\prime}$ and $Q \cup Q^{\prime}$ is an emerald.

Proof. If $M / e$ is not 3-connected then Bixby's Theorem 3.3 implies that $c o(M \backslash e)$ is 3-connected. Since $M$ is 1-irreducible, co $(M \backslash e) \neq M \backslash e$ and $e$ belongs to a triad of $M$. Suppose that $M / e$ is 3-connected. Denoting by $f$ the element in $T^{*}-Q$, we have si $(M /\{e, f\})$ is 3-connected. If $\operatorname{si}(M /\{e, f\}) \neq$ $M /\{e, f\}$, there is a square $Q^{\prime}$ of $M$ containing $e$ and $f$. By orthogonality with $T^{*}$, we have $\left|Q \cap Q^{\prime}\right|=2$ and then $T^{*}$ is contained $Q \cup Q^{\prime}$. Because of Lemma 4.4, we have $Q \cup Q^{\prime}$ is an emerald. If $s i(M /\{e, f\})=M /\{e, f\}$, $M / f$ is 3 -connected. Consequently, there is a square $Q^{\prime}$ of $M$ containing $f$ and avoiding $e$, and so $T^{*} \subseteq Q \cup Q^{\prime}$. We have $\left|Q \cap Q^{\prime}\right|=1$ or, because of Lemma 4.4, $Q \cup Q^{\prime}$ is an emerald.

Lemma 5.2. Let $Q$ be a square of $M$ such that $Q \nsubseteq \mathcal{S}$. Let $T_{1}^{*}$ and $T_{2}^{*}$ be distinct triads of $M$. Then $T_{1}^{*} \cap T_{2}^{*} \cap Q=\emptyset$.

Proof. Suppose, by contradiction, that $T_{1}^{*} \cap T_{2}^{*} \cap Q \neq \emptyset$ for some triads $T_{1}^{*}$ and $T_{2}^{*}$ of $M$. Denoting by $g$ the element in $T_{1}^{*} \cap T_{2}^{*} \cap Q$. Since $Q \nsubseteq \mathcal{S}, Q$ is the unique square of $M$ containing $g$ because of Lemma 4.4. Denote by $T_{i}^{*}=\left\{g, f_{i}, g_{i}\right\}$, for $i \in\{1,2\}$, where $Q=\left\{e, f_{1}, f_{2}, g\right\}$ and $e \notin T_{1}^{*} \cup T_{2}^{*}$.

Sub-lemma 5.2.1. M/e is not 3-connected.

Proof. Suppose $M / e$ is 3-connected. Since $Q \nsubseteq \mathcal{S}$, Lemma 5.1 implies that there are squares $Q_{1}$ and $Q_{2}$ such that $T_{i}^{*} \subseteq Q \cup Q_{i}$ and $Q \cap Q_{i}=$ $\left\{f_{i}\right\}$, for $i \in\{1,2\}$. Therefore $T_{i}^{*}$ is the unique triad that contains $f_{i}$ and co $\left(M \backslash f_{i}\right)=M \backslash f_{i} / g$ is triangle-free, so is not 3-connected because $M$ is 3irreducible. As $c o\left(M / e \backslash f_{1}\right)=M /\{e, g\} \backslash f_{1}$ is 3-connected, we have $e$ is in a series class of $M \backslash f_{1} / g$; a contradiction. 
Sub-lemma 5.2.2. There is a triad $T_{3}^{*}$ such that $T_{3}^{*} \cap Q=\{e, g\}$.

Proof. As $M / e$ is not 3-connected, Lemma 5.1 implies that there is a triad $T_{3}^{*}$ that contains $e$. If $g \notin T_{3}^{*}$ then its contains $f_{i}$ for some $i \in\{1,2\}$. Suppose, without lost of generality, that $f_{1} \in T_{3}^{*}$. Since $\left\{f_{1}\right\}=T_{1}^{*} \cap T_{3}^{*} \cap Q$ and $Q \nsubseteq \mathcal{S}$, we have that $Q$ is the unique square containing $f_{1}$ and then $\left\{f_{2}\right\}=Q-\left(T_{1}^{*} \cup T_{3}^{*}\right)$ plays the role of $e$ in the previous sub-lemma. Hence $M / f_{2}=\operatorname{si}\left(M / f_{2}\right)$ is not 3 -connected and dual form of Tutte's Triangle Lemma 3.4 implies that $M / g_{2}$ and $M / g$ are both 3-connected. Therefore $M$ has a square $Q^{\prime}$ that contains $\left\{f_{2}, g_{2}\right\}$ and $T_{2}^{*}$ is the unique triad that contains $f_{2}$. As $M$ is 3 -irreducible, there is a square $Q^{\prime \prime}$ that contains $g_{2}$ and avoids $f_{2}$. By orthogonality with $T_{2}^{*}$, we have $g \in Q$; a contradiction. Therefore $T_{3}^{*} \cap Q=\{e, g\}$.

Denote by $f_{3}$ the element in $T_{3}^{*}-Q$. We have $M / f_{3}$ and $M / g$ are both 3 -connected, because of the dual form of Tutte's Triangle Lemma. There is a square $Q^{\prime}$ of $M$ such that $f_{3} \in Q^{\prime}$ and Lemma 4.4 implies that $\{e\}=$ $Q \cap Q^{\prime}$. Therefore $T_{3}^{*}$ is the unique triad containing $e$ and $c o(M \backslash e)=M \backslash e / f_{3}$ is 3 -connected. There is a square $Q^{\prime \prime}$ containing $f_{3}$ and avoiding $e$. By orthogonality with $T_{3}^{*}$ we have $g \in Q^{\prime \prime}$; a contradiction.

Lemma 5.3. If $Q$ is a square of $M$ such that $Q \cap T_{1}^{*} \cap T_{2}^{*}=\varnothing$ for every pair of distinct triads $T_{1}^{*}$ and $T_{2}^{*}$ of $M$ then $Q \subseteq \mathcal{S}$. As consequence, every square of $M$ is contained in $\mathcal{S}$.

Proof. Because of Theorem 3.1, there are triads $T_{i}^{*}=\left\{e_{i}, f_{i}, g_{i}\right\}$, for $i \in$ $\{1,2\}$, such that $Q=\left\{f_{1}, g_{1}, f_{2}, g_{2}\right\}$ and $\left\{e_{i}\right\}=T_{i}^{*}-Q$. By hypothesis, they are the only triads intersecting $Q$. We can assume $M / f_{i} 3$-connected.

Because of Bixby's Theorem 3.3, we have si $\left(M / f_{i} / e_{j}\right)$ is 3-connected for $\{i, j\}=\{1,2\}$. If si $\left(M / f_{i} / e_{j}\right) \neq M / f_{i} / e_{j}$, there is a square $Q^{\prime}$ that contains $\left\{f_{i}, e_{j}\right\}$. In this case, $Q \cup Q^{\prime}$ is an emerald, because of Lemma 4.4, and $Q \subseteq \mathcal{S}$. Suppose $M / f_{i} / e_{j}$ is 3-connected, then $M / e_{j}$ is also 3-connected and, by 2-irreducibility of $M$, there is a square $Q^{\prime}$ containing $e_{j}$. If $e_{1}=e_{2}$, $Q \cup Q^{\prime}$ is an emerald and hence $Q \subseteq \mathcal{S}$. If $e_{1} \neq e_{2}$, Lemma 5.1 implies that there are squares $Q_{1}$ and $Q_{2}$ such that $T_{i}^{*} \subseteq Q \cup Q_{i}$, for $i \in\{1,2\}$. We can assume that $\left|Q \cap Q_{i}\right|=1$, otherwise $Q \cup Q_{i}$ is an emerald. Because of Lemma 4.1, co $\left(M \backslash\left(Q \cap Q_{i}\right)\right)=M \backslash\left(Q \cap Q_{i}\right) / e_{i}$ is 3-connected. Since $M$ is 3 -irreducible, there are squares $Q_{1}^{\prime}$ and $Q_{2}^{\prime}$ such that $Q_{i}^{\prime}$ contains $e_{i}$ and avoid the element in $Q \cap Q_{i}$. Thus $T_{i}^{*} \cap Q_{i}^{\prime}=T_{i}^{*}-Q \cap Q_{i}$. If $\left|Q_{i} \cap Q_{i}^{\prime}\right|=2$, Lemma 
4.4 implies that $Q_{i} \cup Q_{i}^{\prime}$ is an emerald. If $Q_{i} \cap Q_{i}^{\prime}=\left\{e_{i}\right\}$ and $T_{i}^{*}$ is the unique triad that contains $e_{i}$, then $Q \cup Q_{i} \cup Q_{i}^{\prime}$ is a pure diamond with nucleus $T_{i}^{*}$. Otherwise, $Q_{i} \cup Q_{i}^{\prime}$ is a sapphire with nucleus $e_{i}$.

Lemma 5.4. Every triad of $M$ is contained in $\mathcal{S}$.

Proof. Suppose the result fails. Let $T^{*}=\{e, f, g\}$ be a triad of $M$ such that $T^{*} \nsubseteq \mathcal{S}$. Then there is a element in $T^{*}$, say $e$, such that $M / e$ is not 3-connected. By dual form of Tutte's Triangle Lemma $3.4 \mathrm{M} / \mathrm{f}$ and $\mathrm{M} / \mathrm{g}$ are both 3 -connected. There is a square $Q$ of $M$ containing $f$ and $g$, and then $\{f, g\} \subseteq \mathcal{S}$. Because of Lemma 4.5, $\{f, g\}$ does not intersect a pure diamond. Lemma 4.3 implies that $\{f, g\}$ does not intersect any emerald. Suppose $\{f, g\}$ intersects a sapphire $S$. Since $M / e$ is not 3-connected, $S$ is forced and $e \in c l_{M}^{*}(S)$, we have $M$ is not 3-connected; a contradiction with Lemma 3.4.

Lemma 5.5. Every element of $M$ belongs to a triad.

Proof. Suppose, by contradiction, that there is no triad of $M$ containing $e$. As $M$ is 1-irreducible, Theorem 3.3 implies that $s i(M / e)=M / e$ is 3 connected. Thus $e$ belongs to a square $Q$ of $M$. By Lemma 5.3 we have $e \in \mathcal{S}$ and so, by the fact of that there is no triad that contains $e$, we can suppose $e$ belongs to a sapphire $S=Q \cup Q^{\prime}$ and there are triads $T_{1}^{*}$ and $T_{2}^{*}$ contained in $S$ such the $e \notin T_{1}^{*} \cup T_{2}^{*}$. Denoting by $F=S-e, F$ is a forced set of $M$ and Lemma 3.15 implies that $c o(M \backslash e)=M \backslash e$ is 3-connected; a contradiction.

Lemmas 5.2 to 5.5 establish that each element of $M$ belongs to a triad contained in union of sapphires, emeralds and pure diamonds. Next lemma improves this result.

Lemma 5.6. If $T^{*}$ is a triad of $M$ then we have 3 possibilities:

i) $T^{*}$ is contained in an emerald; or

ii) $T^{*}$ is a nucleus of a pure diamond; or

iii) there is a sapphire containing $T^{*}$ such that its nucleus is an element of $T^{*}$.

Proof. Because of Lemma 4.3, every triad that intersects an emerald is contained in it. Suppose that $T^{*}$ intersects a pure diamond, but is neither a nucleus of a pure diamond nor intersects an emerald. Then Lemma $4.5 \mathrm{im}-$ plies that $T^{*}$ contains a nucleus of a sapphire. 
Suppose that $T^{*}$ is a triad of $M$ such that $T^{*}$ is not a nucleus of pure diamond, do not contains a nucleus of a sapphire and do not intersects an emerald. Then each element of $T^{*}$ is contained in a sapphire $S$. So, there are distinct squares $Q_{1}$ and $Q_{2}$ such that $\left|Q_{1} \cap Q_{2}\right|=1$ and $T^{*}$ is the unique triad that contains $Q_{1} \cap Q_{2}$. Denote by $\{f\}=Q_{1} \cap Q_{2}$. Lemma 4.1 implies that $c o(M \backslash f)$ is 3 -connected. Since $M$ is 3-irreducible, there is a square $Q_{3}$ such that $Q_{3} \cap\left(Q_{1} \cup Q_{2}\right)=T^{*}-\{f\}$. Therefore $T^{*}$ is a nucleus of a pure diamond or contains a nucleus of a sapphire; a contradiction.

Remembering, $\mathcal{F}$ denotes the class of triangle-free 3 -connected matroids. To conclude the demonstration of Theorem 1.4, it remains to show:

Lemma 5.7. Let $M$ be a semi-binary triangle-free 3-connected matroid with at least 11 elements. Then $M$ is 2-minor-irreducible if and only if is 123irreducible.

Proof. It is sufficient to prove that if $M$ is 123-irreducible then it does not have $N$-minor in $\mathcal{F}$ with $1 \leq|E(M)|-|E(N)| \leq 2$. If $M$ is 123-irreducible, Lemmas 5.5 and 5.6 implies that $M \backslash\{e, f\} \notin \mathcal{F}$ and $M /\{e, f\} \notin \mathcal{F}$, for each 2-subset $\{e, f\}$ of $E(M)$. Suppose $N<M$ with $1 \leq|E(M)|-|E(N)| \leq 2$ and $N \in \mathcal{F}$, for $M$ semi-binary 123-irreducible with at least 11 elements. Then there is a triad $T^{*}$ and a 2-subset $\{e, f\}$ of $T^{*}$ such that $N \simeq M \backslash e / f$. Since $N$ is triangle-free, $T^{*}$ is neither a nucleus of a pure diamond nor is contained in an emerald. Therefore $T^{*}$ contains a nucleus of a sapphire and so $N$ has a triangle; a contradiction.

\section{Proof of Theorem 1.5: Diamantic and totally triangular matroids}

In the first part of this section we give a simple way to check that $P_{7}$ (Figure 8) is the totally triangular matroid with fewest triangles. Then, we define an extension operation for 3-connected matroids, called triangulation around a triad, which is a process of 'placing a triangle around a triad'. In the end, we apply the triangulation around a triad on diamantic matroids to get an associated totally triangular matroid for each of them. 


\subsection{Totally triangular matroid with fewest triangles.}

Tutte's Wheels and Whirls Theorem [17] implies that 3-connected binary matroids with at least 4 elements have $M\left(W_{3}\right)$-minor, since it avoids $U_{2,4^{-}}$ minor. There are only two 3-connected binary matroids with 7 elements: the Fano matroid $F_{7}$ is the binary totally triangular matroid with fewest triangles and $F_{7}^{*}$ is the smallest binary triangle-free 3 -connected matroid. Let $M$ be a totally triangular matroid that is representable over some field and suppose that $M$ is non-binary with at least 4 elements. Suppose $|E(M)| \leq 7$. Because of Theorem 2.1 of [11], we can assume that $M$ has no $U_{2,5}$-minor. The main result of [15] implies that $M$ is ternary. Theorem 2 of [1] implies that $M \simeq P_{7}$ or $M$ has a minor isomorphic to a 3 -wheel.



Figure 8: Geometric representation of $P_{7}$.

For convenience, in this subsection we call a 3-connected matroid by strictly triangular matroid if each of its elements belongs to at least 2 triangles and every pair of triangles intersects in at most one element. So, a totally triangular matroid is a triad-free strictly triangular matroid.

Lemma 6.1. Up to isomorphism:

i) the 3-wheel $M\left(W_{3}\right)$ is the only strictly triangular matroid with 4 triangles; and

ii) $P_{7}$ is the only strictly triangular matroid with 5 triangles.

As consequence, $P_{7}$ is the totally triangular matroid with fewest triangles.

Proof. (i) Let $M$ be a strictly triangular matroid with 4 triangles, $T_{i}$ for $i=1,2,3$ and 4 . We can assume that $T_{1}=\{1,2,3\}$ and that $i \in T_{i+1}$, for 
each $i \in\{1,2,3\}$. Since $\left|T_{1} \cap T_{2}\right|=1$, we can suppose that $T_{2}=\{1,4,5\}$, and so $|E(M)| \geq 5$, and that $T_{2} \cap T_{3} \neq \emptyset$. Without loss of generality, we can take $T_{3}=\{2,4,6\}$. Since each element belongs to at least 2 triangles, we have $T_{4}=\{3,5,6\}$. Since $r(M)=3$, a geometric representation for $M$ can be obtained by drawing four 3-point-lines, representing the triangles of $M$, and identifying the common points of these triangles.

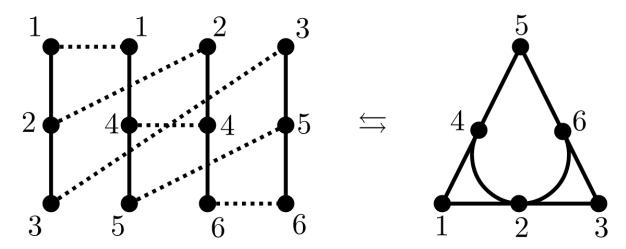

Figure 9: Geometric representation of 3-wheel, the smallest strictly triangular matroid.

(ii) Suppose that $M$ is a strictly triangular matroid with 5 triangles, $T_{i}$ for $i=1,2,3,4$ and 5 . By previous considerations, we can assume that $|E(M)| \geq 6, T_{1}=\{1,2,3\}, i \in T_{i+1}$ for each $i \in\{1,2,3\}, T_{2}=\{1,4,5\}$ and $T_{3}=\{2,4,6\}$. We have that $T_{4} \cap T_{2} \neq \emptyset$ or $T_{4} \cap T_{3} \neq \emptyset$. By symmetry, we can suppose $T_{4} \cap T_{2} \neq \emptyset$. If $4 \notin T_{4}$ then $T_{4}=\{3,5, x\}$ for a new element $x$, otherwise $x=6$ and it is impossible to properly form the triangle $T_{5}$. Hence $T_{4}=\{3,5,7\}$ and this forces $T_{5}=\{1,6,7\}$. If $4 \in T_{4}$ then $T_{4}=\{3,4,7\}$ and $T_{5}=\{5,6,7\}$. It is not difficult to see that in both case $M$ is isomorphic to $P_{7}$.

\subsection{Triangulation around a triad.}

The bijection referred in Theorem 1.5 is based on a construction via generalized parallel connection. For definitions, notations and properties on generalized parallel connection see Oxley's book [12], sections 11.4 and 11.5. Let $M$ be a 3-connected matroid, $T^{*}$ a triad and $Y$ a triangle of $M$. We say that $Y$ is around $T^{*}$ in $M$ if $M \mid\left(T^{*} \cup Y\right)$ is isomorphic to a cycle matroid of a 3-wheel.

Denote by $K_{5} \backslash e$ the complete graph on five vertices with one edge deleted. We call $M^{*}\left(K_{5} \backslash e\right)$ by prism matroid. The prism matroid has two disjoint triangles, say $T$ and $T^{\prime}$. If $M$ is a 3 -connected matroid and $T^{*}$ is 
a triad of $M$, we can identify a triangle of $M^{*}\left(K_{5} \backslash e\right)$ with $T^{*}$, say $T=$ $T^{*}$, such that $E\left(M^{*}\left(K_{5} \backslash e\right)\right) \cap E(M)=T^{*}$. Let $P_{T^{*}}\left(M^{*}\left(K_{5} \backslash e\right), M^{*}\right)$ be the generalized parallel connection of $M^{*}\left(K_{5} \backslash e\right)$ and $M^{*}$ across $T^{*}$. We have that $P_{T^{*}}\left(M^{*}\left(K_{5} \backslash e\right), M^{*}\right)$ is a 3-connected matroid with ground set $E(M) \cup E\left(M^{*}\left(K_{5} \backslash e\right)\right)$ and rank $r\left(M^{*}\right)+3$. The simplification of its dual, si $\left(P_{T^{*}}^{*}\left(M^{*}\left(K_{5} \backslash e\right), M^{*}\right) / T^{*}\right)$, is a 3-connected matroid with rank $r(M)$. It is possible to re-label the elements of $T^{\prime}$, in $\operatorname{si}\left(P_{T^{*}}^{*}\left(M^{*}\left(K_{5} \backslash e\right), M^{*}\right) / T^{*}\right)$, in order to obtain a 3-connected matroid $N$ with ground set $E(N)=E(M) \cup$ $Y$, where $Y=E\left(M^{*}\left(K_{5} \backslash e\right)\right)-\left(T^{*} \cup T^{\prime}\right)$, such that $N \mid E(M)=M$ and $N \mid\left(T^{*} \cup Y\right)$ is isomorphic to a 3 -wheel having $Y$ as its rim.

Note that $N \backslash T^{*}$ is isomorphic to the matroid obtained from $M$ after a simplification of $Y-\Delta$ operation along the triad $T^{*}: \quad N \backslash T^{*}=$ si $\left(P_{T^{*}}^{*}\left(W_{3}, M^{*}\right) / T^{*}\right)$ where $W_{3}=M^{*}\left(K_{5} \backslash e\right) / T^{\prime}$ is a 3 -wheel. Therefore

$$
N=\operatorname{si}\left(P_{Y}\left(W_{3}^{*}, P_{T^{*}}^{*}\left(W_{3}, M^{*}\right) / T^{*}\right)\right)
$$

which is a more descriptive way to obtain $N$ from $M$. When $M$ has a triangle $T$ intersecting $T^{*}$, there is a parallel class in $P_{Y}\left(W_{3}^{*}, P_{T^{*}}^{*}\left(W_{3}, M^{*}\right) / T^{*}\right)$ containing the element in $T-T^{*}$ and just one element of $Y$. In this case, we treat the class representative in $N$ with the label coming from $E(M)$ or $E\left(W_{3}\right)$, whichever is more convenient for each situation. By bearing this caveat in mind, if $M$ has a triangle around $T^{*}$ then $N=M$ and $N \mid\left(T^{*} \cup Y\right)=W_{3}^{*}$.

Let $M$ be a 3-connected matroid and $T^{*}$ a triad of $M$. Take $M^{*}\left(K_{5} \backslash e\right)$ a prism matroid such that $E\left(M^{*}\left(K_{5} \backslash e\right)\right) \cap E(M)=T^{*}$ is a triangle of $M^{*}\left(K_{5} \backslash e\right)$. Denote by $W_{3}=M^{*}\left(K_{5} \backslash e\right) / T^{*}$ the 3 -wheel where $T^{* *}$ is the triad of $M\left(K_{5} \backslash e\right)$ disjunct from $T^{*}$. We define the triangulation around $T^{*}$ as the matroid

$$
\mathbf{\Delta}_{T^{*}}(M)=\operatorname{si}\left[P_{Y}\left(W_{3}^{*}, P_{T^{*}}^{*}\left(W_{3}, M^{*}\right) / T^{*}\right)\right]
$$

which can be obtained from si $\left(P_{T^{*}}^{*}\left(M^{*}\left(K_{5} \backslash e\right), M^{*}\right) / T^{*}\right)$ exchanging conveniently the labels of elements of $T^{*}$ to labels of $T^{*}$.

Example 6.2. The Figure 10 illustrates what happens after triangulation around a triad in the cube graph $D_{6}$. In dashed lines, the triangle placed around the inner triad. 


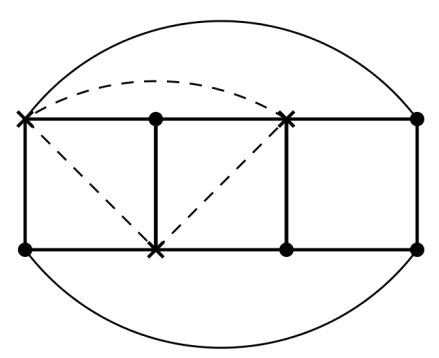

Figure 10: Triads destroyed on triangulation around a triad in $D_{6}$ are marked.

The following results derive from the properties of the generalized parallel connection and usual arguments in matroids. We have omitted their respective proofs.

Lemma 6.3. Let $T^{*}$ be a triad of a 3-connected matroid $M$ and $Y$ the triangle around $T^{*}$ in $\boldsymbol{\Delta}_{T^{*}}(M)$. Then:

i) If $N$ is a 3-connected matroid with ground set $E=E(M) \cup Y$ such that $N \mid E(M)=M, T^{*}$ is a triad of $N$ and $Y$ is a triangle around $T^{*}$ on $N$, then $N \simeq \mathbf{\Delta}_{T^{*}}(M)$

ii) $\mathbf{\Delta}_{T^{*}}(M) \backslash T^{*}$ is 3-connected and $r\left(\boldsymbol{\Lambda}_{T^{*}}(M) \backslash T^{*}\right)=r(M)-1$;

iii) If $T^{*}$ is a nucleus of a pure diamond of $M$, each element of $Y$ belongs to at least two triangles of $\boldsymbol{\Delta}_{T^{*}}(M) \backslash T^{*}$;

iv) If a subset $X \subseteq Y$ do not intersects any triad of $M$ then $M \backslash X$ is 3connected;

v) Suppose for each triangle $T$ of $M$ that intersects $T^{*}$ we have $T-T^{*}$ do not belongs to any triad of $M$ (equivalently, $M \backslash\left(T-T^{*}\right)$ is 3-connected). Then the triads of $\mathbf{\Delta}_{T^{*}}(M)$ are $T^{*}$ and triads of $M$ that do not intersects $T^{*}$. Triads of $M$ that intersects $T^{*}$, different of it self, are destroyed;

Lemma 6.4. Let $M$ be a 3-connected matroid with $T^{*}$ and $T^{*}$ different triads of $M$. Then $\boldsymbol{\Delta}_{T^{*}}(M)=\boldsymbol{\Delta}_{T^{* *}}(M)$ if and only if $T^{*} \cup T^{*}$ is a pure emerald of M. Moreover, if $T^{*} \cup T^{* *}$ is a non-pure emerald then $\boldsymbol{\Delta}_{T^{*}}(M)$ is non-semibinary and $\mathbf{\Delta}_{T^{*}}\left(\boldsymbol{\Delta}_{T^{* *}}(M)\right)$ has triangles $T$ and $T^{\prime}$ such that $\left|T \cap T^{\prime}\right|=2$.

6.3. One-to-one correspondence between diamantic and totally triangular matroids.

Let $M$ be a diamantic matroid with $n$ triads. Fix an order for the family of triads of $M$, say $\left\{T_{1}^{*}, T_{2}^{*}, \ldots, T_{n}^{*}\right\}$. For each $k \in\{1,2, \ldots, n\}$, we denote 
by $W_{3}^{k}$ a copy of a 3 -wheel with ground set $E\left(W_{3}^{k}\right)=T_{k}^{*} \cup Y_{k}$ in which $T_{k}^{*}$ is a spokes set and $Y_{k}$ a rim of $W_{3}^{k}$, such that $Y_{k} \cap\left(E(M) \cup\left(\cup_{i \neq k} Y_{i}\right)\right)=\emptyset$. Denote by $M_{1}=\mathbf{\Delta}_{T_{1}^{*}}(M)$ and $M_{k}=\mathbf{\Delta}_{T_{k}^{*}}\left(M_{k-1}\right)$, for each $k \in\{2, \ldots, n\}$. Consider the following sets

$\widehat{Y}_{k}=Y_{k}-\left\{y \in Y_{k}: y\right.$ is in a parallel class of $\left.P_{Y_{k}}\left(W_{3}^{k}, P_{T_{k}^{*}}^{*}\left(W_{3}^{k *}, M_{k-1}^{*}\right) / T_{k}^{*}\right)\right\}$ and take $\widehat{Y}_{1}=Y_{1}$. Then $E\left(M_{k}\right)=E(M) \cup \widehat{Y}_{1} \cup \cdots \cup \widehat{Y}_{k}$.

Lemma 6.5. For each $k \in\{1,2, \ldots, n\}, M_{k}$ is a 3-connected matroid such that:

i) $M_{k} \backslash \widehat{Y}_{k}=M_{k-1}$, and so $M_{k} \backslash\left(\widehat{Y}_{1} \cup \cdots \cup \widehat{Y}_{k}\right)=M$;

ii) $r\left(M_{k}\right)=r(M)$;

iii) $M_{k} \backslash\left(T_{1}^{*} \cup \cdots \cup T_{k}^{*}\right)$ is a 3-connected matroid with rank $r(M)-k$;

iv) each element of $\widehat{Y}_{1} \cup \cdots \cup \widehat{Y}_{k}$ belongs to at least two triangles of $M_{k} \backslash\left(T_{1}^{*} \cup \cdots \cup T_{k}^{*}\right)$;

v) the triads of $M_{k}$ are the same triads of $M$.

As consequence, $M_{n} \backslash\left(T_{1}^{*} \cup \cdots \cup T_{n}^{*}\right)$ is a totally triangular matroid with $\operatorname{rank} r(M)-n$.

Proof. Items $(i),(i i)$ and $(i i i)$ are consequence of Lemma 6.3. Item $(i v)$ : suppose valid for $k-1$. Take $y \in\left(\widehat{Y}_{1} \cup \cdots \cup \widehat{Y}_{k}\right)$. If $y \in \widehat{Y}_{j}$ for $j<k$ then $y$ belongs to at least two triangles of $M_{k-1}$, and so of $M_{k}$. Otherwise $y \in \widehat{Y}_{k}$ and we can apply the same arguments as Lemma 6.3 (iii). Item $(v)$ is consequence of Lemma $6.3(v)$, since each element of $M$ belongs to a unique triad.

It is straightforward to see that $M_{n}$ does not depend on the order of the triads.

Denote by $\mathcal{D}^{n}$ the family of diamantic matroids with $n$ triads and $\mathcal{T}^{n}$ the family of totally triangular matroids with $n$ triangles, for $n \geq 5$ (Lemma 6.1). Previous lemma implies that $b: \mathcal{D}^{n} \longrightarrow \mathcal{T}^{n}$ such that

$$
b(M)=M_{n} \backslash\left(T_{1}^{*} \cup \cdots \cup T_{n}^{*}\right)
$$

is an injective function, up to isomorphism. The construction of $b$ reveals how to obtain its inverse. From a totally triangular matroid $N$, the process for obtaining the associated diamantic matroid is simpler as it involves general parallel connection directly. 
Let $N$ be a totally triangular matroid. Denote by $\left\{Y_{1}, Y_{2}, \ldots, Y_{n}\right\}$ the family of triangles of $N$. For each $k \in\{1,2, \ldots, n\}$, take $W_{3}^{k}$ a copy of a 3-wheel in which $T_{k}^{*}$ is a spokes set and $Y_{k}$ a rim of $W_{3}^{k}$, such that $T_{k}^{*} \cap$ $\left(E(N) \cup\left(\cup_{i \neq k} T_{i}^{*}\right)\right)=\emptyset$. We denote by $N^{1}=P_{Y_{1}}\left(W_{3}^{1}, N\right)$ and, for $1<k \leq$ $n$, we denote by $N^{k}=P_{Y_{k}}\left(W_{3}^{k}, N^{k-1}\right)$. We have $Y_{k}$ a triangle of $N^{k-1}$ and, for every $k \in\{1,2, \ldots, n\}, T_{k}^{*}$ is a triad of both $N^{k}$ and $N^{k} \backslash Y_{k}$. Moreover, $N^{k} \backslash T_{k}^{*}=N^{k-1}$ and $N^{k} \backslash\left(T_{1}^{*} \cup \cdots \cup T_{k}^{*}\right)=N$.

Lemma 6.6. $N^{k} \backslash\left(Y_{1} \cup \cdots \cup Y_{k}\right)$ is a 3-connected matroid having $T_{1}^{*}, T_{2}^{*}, \ldots$, $T_{k}^{*}$ as triads and rank $r\left(N^{k} \backslash\left(Y_{1} \cup \cdots \cup Y_{k}\right)\right)=r(N)+k$, for each $k \in$ $\{1,2, \ldots, n\}$. Moreover, $N^{n} \backslash\left(Y_{1} \cup \cdots \cup Y_{n}\right)$ is a diamantic matroid.

Proof. For every $k$, we have that $N^{k}=P_{Y_{k}}\left(W_{3}^{k}, N^{k-1}\right)$ is 3-connected and $T_{k}^{*}$ is the unique cocircuit of $N^{k}$ containing in $E\left(W_{3}^{k}\right)$. Take $T^{*}$ a triad of $N^{k}=P_{Y_{k}}\left(W_{3}^{k}, N^{k-1}\right)$. Then $T^{*}=T_{k}^{*}$ or $T^{*} \cap E\left(W_{3}^{k}\right)=\emptyset$. If $T^{*} \neq T_{k}^{*}$ then $T^{*}$ is a triad of $N^{k-1} / Y_{k}$ and so $T^{*}$ is a triad of $N^{k-1}$. It follows by induction that $T_{1}^{*}, T_{2}^{*}, \ldots, T_{k}^{*}$ are the triads of $N^{k}$. By Lemma 6.3 (iv), we have that $N^{k} \backslash Y_{k}$ is 3-connected. Denote by $\widehat{Y}_{1}=Y_{1}$ and, for $k>1$, $\widehat{Y}_{k}=Y_{k}-\left(Y_{1} \cup \cdots \cup Y_{k-1}\right)$. As $N^{k}$ is a 3-connected matroid and $Y_{k}$ is a triangle around $T_{k}^{*}$ in $N^{k}$ such that $Y_{k}$ do not intersects any triad of $N^{k}$, Lemma 6.3 implies that $N^{k} \backslash \widehat{Y}_{k}$ is 3-connected. We have that $Y_{k-1}$ is a triangle around $T_{k-1}^{*}$ on $N^{k} \backslash \widehat{Y}_{k}$. Then $N^{k} \backslash \widehat{Y}_{k} \backslash \widehat{Y_{k-1}}$ is 3-connected. Continuing with this precedence, we obtain that $N^{k} \backslash\left(Y_{1} \cup \cdots \cup Y_{k}\right)$ is 3-connected. Elimination circuit axiom ensures that each triad of $N^{n} \backslash\left(Y_{1} \cup \cdots \cup Y_{n}\right)$ is a nucleus of a pure diamond. Since each pair of triangles of $N$ intersects in at most one element, $N^{n} \backslash\left(Y_{1} \cup \cdots \cup Y_{n}\right)$ is emerald-free because of Lemma 6.4. Therefore, $N^{n} \backslash\left(Y_{1} \cup \cdots \cup Y_{n}\right)$ is a diamantic matroid.

It follows from properties of generalized parallel connection that $N^{n}$ does not depend on the order of the triangles. Therefore, we have $\sharp: \mathcal{T}^{n} \longrightarrow \mathcal{D}^{n}$ such that

$$
\sharp(N)=N^{n} \backslash\left(Y_{1} \cup \cdots \cup Y_{n}\right)
$$

is injective, up to isomorphism. To conclude the proof of Theorem 1.5, it remains to be seen that $\sharp=b^{-1}$.

Lemma 6.7. For $M \in \mathcal{D}^{n}, \sharp(b(M))=M$.

Proof. Take $M \in \mathcal{D}^{n}$. Then

$$
\begin{aligned}
\sharp(b(M)) & =(b(M))^{n} \backslash\left(Y_{1} \cup \cdots \cup Y_{n}\right) \\
& =\left(M_{n} \backslash\left(T_{1}^{*} \cup \cdots \cup T_{n}^{*}\right)\right)^{n} \backslash\left(Y_{1} \cup \cdots \cup Y_{n}\right)
\end{aligned}
$$


Note that

$$
\begin{aligned}
\left(M_{n} \backslash\left(T_{1}^{*} \cup \cdots \cup T_{n}^{*}\right)\right)^{1} & =P_{Y_{1}}\left(W_{3}^{1}, M_{n} \backslash\left(T_{1}^{*} \cup \cdots \cup T_{n}^{*}\right)\right) \\
& =M_{n} \backslash\left(T_{2}^{*} \cup \cdots \cup T_{n}^{*}\right)
\end{aligned}
$$

and

$$
\begin{aligned}
\left(M_{n} \backslash\left(T_{1}^{*} \cup \cdots \cup T_{n}^{*}\right)\right)^{2} & =P_{Y_{2}}\left(W_{3}^{2}, M_{n} \backslash\left(T_{2}^{*} \cup \cdots \cup T_{n}^{*}\right)\right) \\
& =M_{n} \backslash\left(T_{3}^{*} \cup \cdots \cup T_{n}^{*}\right)
\end{aligned}
$$

and so on until

$$
\left(M_{n} \backslash\left(T_{1}^{*} \cup \cdots \cup T_{n}^{*}\right)\right)^{n}=M_{n}
$$

Therefore

$$
\sharp(b(M))=M_{n} \backslash\left(Y_{1} \cup \cdots \cup Y_{n}\right)=M .
$$

Example 6.8. In Lemma 6.1, we prove that $P_{7}$ is the totally triangular matroid with fewest triangles. Consequently $b^{-1}\left(P_{7}\right)$ is the smallest diamantic matroid, with 15 elements and rank 8.

Example 6.9. For $n \geq 4$, let $M\left(L_{n}\right)$ and $M\left(\mathcal{L}_{n}\right)$ be the ladder and Möbius ladder, respectively. Since $M^{*}\left(L_{n}\right)$ and $M^{*}\left(\mathcal{L}_{n}\right)$ are two non-isomorphic totally triangular matroids, $b^{-1}\left(M^{*}\left(L_{n}\right)\right)$ and $b^{-1}\left(M^{*}\left(\mathcal{L}_{n}\right)\right)$ are two nonisomorphic diamantic matroids with $6 n$ elements and rank $3 n+1$.

\section{Proof of Theorem 1.6: Emeralds in binary matroids}

We separate the proof of Theorem 1.6 into two parts. In the first part of this section we prove that, up to isomorphism, the graphic matroid $M\left(K_{3, n}\right)$, for $n \geq 3$, is the only rank $n+23$-connected matroid in which each pair of elements is in a pure emerald. The proof follows a script close to that given in Section 2 of Oxley, Pfeil, Semple and Whittle [14]. In the second part we prove that if the ground set of a 3 -connected binary matroid $M$ can be write as an union of emeralds then $M$ is isomorphic to $M\left(K_{3, n}\right)$. 


\subsection{Proof of Theorem 1.6: (ii) implies (iv)}

We need the concept of weak map. Let $M$ and $N$ be two matroids and let $\omega: E(M) \longrightarrow E(N)$ be a bijection. We say that $\omega$ is a weak map from $M$ to $N$ if for each independent set $I$ in $N$, we have $\omega^{-1}(I)$ is independent in $M$. Equivalently, for every circuit $C$ of $M$, we have $\omega(C)$ contains a circuit of $N$. The following theorem is due to Lucas [7].

Theorem 7.1. Let $\omega: E(M) \longrightarrow E(N)$ be a weak map from a binary matroid $M$ to a matroid $N$. If $r(M)=r(N)$ then $N$ is binary. Moreover, if $N$ is connected then $N \simeq M$.

Here we prove the following result:

Theorem 7.2. Let $M$ be a 3-connected matroid, with $|E(M)| \geq 9$, such that every pair of elements is in a pure emerald. Then $M \simeq M\left(K_{3, n}\right)$ where $n=\frac{|E(M)|}{3}$.

Proof. We can partition $E(M)$ in a disjunct union of triads $E(M)=T_{1}^{*} \cup$ $T_{2}^{*} \cup \cdots \cup T_{n}^{*}$ for some $n \geq 3$ such that $T_{i}^{*} \cup T_{j}^{*}$ is a pure emerald for $1 \leq i<j \leq n$. Furthermore, we can choose the labels of $T_{i}^{*}=\left\{e_{i}, f_{i}, g_{i}\right\}$ so that $\left\{x_{i}, y_{i}, x_{j}, y_{j}\right\}$ is a square of $M$ for each 2-subset $\{x, y\} \subseteq\{e, f, g\}$ and for $1 \leq i<j \leq n$. By orthogonality, for distinct triads $T_{i}^{*}, T_{j}^{*}$ and $T_{k}^{*}$, if $Q$ is a square contained in $T_{i}^{*} \cup T_{j}^{*}$ and $Q^{\prime}$ is a square contained in $T_{j}^{*} \cup T_{k}^{*}$ such that $\left|Q \cap Q^{\prime}\right|=1$, then the symmetric difference $Q \triangle Q^{\prime}$ is a circuit of $M$. Lemma 4.2 implies that $F=T_{1}^{*} \cup T_{2}^{*}$ is a forced set with $r(F)=r^{*}(F)=4$. For each $2<i \leq n, T_{i}^{*} \cap c l\left(T_{1}^{*} \cup \cdots \cup T_{i-1}^{*}\right)=\emptyset$ and then $r\left(T_{1}^{*} \cup \cdots \cup T_{i-1}^{*} \cup T_{i}^{*}\right)=r\left(T_{1}^{*} \cup \cdots \cup T_{i-1}^{*}\right)+1$. Consequently $r(M)=n+2$.

Let $N$ be a matroid with ground set $E(N)=E(M)$ such that $N \simeq$ $M\left(K_{3, n}\right)$ having $\left\{e_{i}, f_{i}, g_{i}\right\}$ as triads and having $\left\{x_{i}, y_{i}, x_{j}, y_{j}\right\}$ as squares for each 2-subset $\{x, y\} \subseteq\{e, f, g\}$ and for $1 \leq i<j \leq n$. The identity map from $E(N)$ to $E(M)$ is a weak map from $M\left(K_{3, n}\right)$ to $M$. As $r\left(M\left(K_{3, n}\right)\right)=n+2$ and $M$ is 3-connected, Theorem 7.1 implies that $M \simeq M\left(K_{3, n}\right)$.

\subsection{Proof of Theorem 1.6: (iii) implies (iv)}

It is known that if $M$ is a 3-connected binary matroid with $6 \leq|E(M)| \leq$ 8 then $M$ is isomorphic to $M\left(W_{3}\right), F_{7}, F_{7}^{*}, A G(3,2), S_{8}$ or $M\left(W_{4}\right)$. All of 
these matroids are well known. Let $M$ be a 3-connected binary matroid with $|E(M)|=9$. If $M$ has no 4 -wheel minor then $M$ is isomorphic to $Z_{4}$ or $Z_{4}^{*}$. If $M$ has a 4 -wheel minor and is non-regular then $M$ is isomorphic to $P_{9}$ or $P_{9}^{*}$. If $M$ is a regular matroid having a 4 -wheel minor then $M$ is isomorphic to $M\left(K_{3,3}\right), M^{*}\left(K_{3,3}\right), M\left(K_{5} \backslash e\right)$ or $M^{*}\left(K_{5} \backslash e\right)$. Matrix representations of $Z_{4}$ and $P_{9}$ can be found in Kingan and Lemos [3].

Theorem 7.3. Let $M$ be a 3-connected binary matroid with at least 9 elements. If each of its elements belongs to an emerald then $M \simeq M\left(K_{3, n}\right)$.

Proof. We can partition $E(M)$ in a disjunct union of triads $T_{1}^{*} \cup T_{2}^{*} \cup \cdots \cup T_{n}^{*}$ for some $n \geq 3$. This implies that every circuit of $M$ has even cardinality. We prove by induction in $n$. For $n=3$ we have that every pair of elements of $M$ is in a pure emerald. Theorem 7.2 implies that $M \simeq M\left(K_{3,3}\right)$.

Suppose $|E(M)|=3 n$ for $n>3$. Choose $T_{1}^{*}$ and $T_{2}^{*}$ triads of $M$ such that $F=T_{1}^{*} \cup T_{2}^{*}$ is an emerald. Lemma 4.3 implies that $M \backslash T_{1}^{*}$ is 3-connected. If each element of $M \backslash T_{1}^{*}$ is in an emerald then $M \backslash T_{1}^{*} \simeq M\left(K_{3, n-1}\right)$, by induction hypothesis. In this case,

$$
M \simeq P_{T}\left(W_{3}, \mathbf{\Delta}_{T^{*}}\left(M\left(K_{3, n-1}\right)\right)\right) \backslash T \simeq M\left(K_{3, n}\right)
$$

where $T^{*}$ is a triad of $M\left(K_{3, n-1}\right), T$ is the triangle around $T^{*}$ in $\boldsymbol{\Delta}_{T^{*}}\left(M\left(K_{3, n-1}\right)\right)$ and $W_{3}$ is a 3 -wheel over $E\left(W_{3}\right)=T \cup T^{* *}$ with $\operatorname{rim} T$ and $E\left(W_{3}\right) \cap E\left(\boldsymbol{\Delta}_{T^{*}}\left(M\left(K_{3, n-1}\right)\right)\right)=T$.

If there are elements of $M \backslash T_{1}^{*}$ that do not belongs to any emerald then these elements are in $T_{2}^{*}$. Each element of $E(M)-F$ belongs to an emerald of $M \backslash T_{1}^{*}$. Since $\{F, E(M)-F\}$ is an exact 3-separation for $M$ with $|F|,|E(M)-F| \geq 6$, there is a binary matroid $N$ with ground set $E(M) \cup T$, unique up to a permutation on the labels of the elements of $T$, such that $N \mid E(M)=M, T$ is a triangle of $N$ such that $r_{N}(F \cup T)=r_{M}(F)$ and $r_{N}((E(M)-F) \cup T)=r_{M}(E(M)-F)$. Moreover, $M=P_{T}\left(M_{1}, M_{2}\right) \backslash T$ is the 3 -sum of $M_{1}$ and $M_{2}$ where $M_{1}=N \mid(F \cup T)$ and $M_{2}=$ $N \mid((E(M)-F) \cup T)$. Furthermore, if $\{X, Y\}$ is a 2-separation of $M_{1}$ then $X=\{x, t\}$ where $x \in F, t \in T$ and $X$ is a parallel class in $M_{1}$. As consequence, si $\left(M_{1}\right)$ is 3 -connected. These properties of $M$ stem from the results on 3-separations due to Seymour [16]. For more direct results, see Proposition 9.3.4 [12] and (4.3) [16]. Every circuit $C$ of $M_{2}$ such that $|C \cap T|=1$ has odd cardinality and then $s i\left(M_{1}\right)=M_{1}$, otherwise $M_{1}$ has a parallel class $\{x, t\}$ where $x \in F$ and $t \in T$. In this case $M=P_{T}\left(M_{1}, M_{2}\right) \backslash T$ has a circuit with odd cardinality, and this is a contradiction. 
As consequence, $M_{1}$ is a 3-connected binary matroid with 9 elements and then

$$
M_{1} \in\left\{M^{*}\left(K_{3,3}\right), M\left(K_{5} \backslash e\right), M^{*}\left(K_{5} \backslash e\right), P_{9}, P_{9}^{*}, Z_{4}\right\} .
$$

It is simple to see that if $M^{\prime} \in\left\{M^{*}\left(K_{3,3}\right), M^{*}\left(K_{5} \backslash e\right), P_{9}, P_{9}^{*}\right\}$ and $T^{\prime}$ is a triangle of $M^{\prime}$ then $M^{\prime} \backslash T^{\prime}$ has at most one square. Consequently, $M_{1} \in\left\{M\left(K_{5} \backslash e\right), Z_{4}\right\}$. By symmetry, any of the four triangles of $Z_{4}$ can be taken to make the generalized parallel connection with $M_{2}$. Since $Z_{4}$ has a circuit $C$ with even cardinality intersecting $T$ in just one element, we have that $P_{T}\left(Z_{4}, M_{2}\right) \backslash T$ has circuits with odd cardinality and then $M$ is not isomorphic to $P_{T}\left(Z_{4}, M_{2}\right) \backslash T$.

Therefore $M_{1} \simeq M\left(K_{5} \backslash e\right)$. Let $T$ be the only triangle of $M\left(K_{5} \backslash e\right)$ that do not intersects any triad. If $T^{\prime}$ is a triangle of $M\left(K_{5} \backslash e\right)$ different from $T$ then $M\left(K_{5} \backslash e\right) \backslash T^{\prime}$ has just one square. We have that $M\left(K_{5} \backslash e\right) \backslash T \simeq$ $M\left(K_{3,2}\right)$. Every circuit $C$ of $M\left(K_{5} \backslash e\right)$ such that $|C \cap T|=1$ has odd cardinality and the triads of $M\left(K_{5} \backslash e\right)$ remains triads in $P_{T}\left(M\left(K_{5} \backslash e\right), M_{2}\right) \backslash T$. Therefore $M_{1}$ is isomorphic to $M\left(K_{5} \backslash e\right)$ having $T_{1}^{*}, T_{2}^{*}$ as triads. So, $M_{1} \backslash T_{1}^{*}$ is a 3 -wheel with ground set $T \cup T_{2}^{*}$ and then

$$
M \backslash T_{1}^{*}=P_{T}\left(M_{1} \backslash T_{1}^{*}, M_{2}\right) \backslash T \simeq \Delta_{T}\left(M_{2}\right)
$$

where $\Delta_{T}\left(M_{2}\right)$ denotes the matroid obtained from $M_{2}$ after $\Delta-Y$ operation on $T$. Consequently, $M \backslash\left(T_{1}^{*} \cup T_{2}^{*}\right)=M_{2} \backslash T$. Due to the uniqueness of $N$, we have $N=P_{T}\left(M_{1}, M_{2}\right)=\mathbf{\Delta}_{T_{1}^{*}}(M)$ and so $N$ is 3 -connected.

Sub-theorem 7.3.1. $M_{2} \backslash T$ is 3-connected.

Proof. Suppose that $\{X, Y\}$ is an exact $k$-separation for $M_{2} \backslash T=$ $M \backslash\left(T_{1}^{*} \cup T_{2}^{*}\right)$ with $k \leq 2$. We have

$$
\begin{aligned}
k & =r_{M \backslash T_{1}^{*}}(X)+r_{M \backslash T_{1}^{*}}^{*}\left(X \cup T_{2}^{*}\right)-2-|X|+1 \\
& =\lambda_{M \backslash T_{1}^{*}}\left(X \cup T_{2}^{*}\right)-\delta\left(X \cup T_{2}^{*}\right)+2
\end{aligned}
$$

where $2 \leq \delta\left(X \cup T_{2}^{*}\right)=r_{M \backslash T_{1}^{*}}\left(X \cup T_{2}^{*}\right)-r_{M \backslash T_{1}^{*}}(X) \leq 3$.

Since $N=\boldsymbol{\Delta}_{T_{1}^{*}}(M)$, we have that $r_{N}^{*}\left(X \cup T_{2}^{*} \cup T\right)=r_{M}^{*}\left(X \cup T_{2}^{*}\right)$.

As $r_{M \backslash T_{1}^{*}}^{*}\left(X \cup T_{2}^{*}\right)=r_{M}^{*}\left(X \cup T_{2}^{*} \cup T_{1}^{*}\right)-2$, there is $s \in\{1,2\}$ such that $r_{M \backslash T_{1}^{*}}^{*}\left(X \cup T_{2}^{*}\right)=r_{M}^{*}\left(X \cup T_{2}^{*}\right)+s-2$.

Hence 


$$
\begin{aligned}
\lambda_{N}\left(X \cup T_{2}^{*} \cup T\right) & =r_{N}\left(X \cup T_{2}^{*} \cup T\right)+r_{N}^{*}\left(X \cup T_{2}^{*} \cup T\right)-\left|\left(X \cup T_{2}^{*} \cup T\right)\right| \\
& =r_{M \backslash T_{1}^{*}}\left(X \cup T_{2}^{*}\right)+r_{M \backslash T_{1}^{*}}^{*}\left(X \cup T_{2}^{*}\right)+2-s-\left|\left(X \cup T_{2}^{*}\right)\right|-3 \\
& =\lambda_{M \backslash T_{1}^{*}}\left(X \cup T_{2}^{*}\right)-s-1 \\
& =k+\delta\left(X \cup T_{2}^{*}\right)-2-s-1 \\
& <k
\end{aligned}
$$

a contradiction, since $N$ is 3 -connected.

Then $M_{2} \backslash T$ is a 3 -connected binary matroid such that each element belongs to an emerald with $\left|E\left(M_{2} \backslash T\right)\right|=3(n-2)$. By the induction hypothesis we have that $M_{2} \backslash T \simeq M\left(K_{3, n-2}\right)$ and so $M_{2} \simeq \mathbf{\Delta}_{T^{*}}\left(M\left(K_{3, n-2}\right)\right)$ for any $T^{*}$ triad of $M\left(K_{3, n-2}\right)$. Therefore

$$
M=P_{T}\left(M_{1}, M_{2}\right) \backslash T \simeq P_{T}\left(M\left(K_{5} \backslash e\right), M\left(K_{3, n-2}\right)\right) \backslash T \simeq M\left(K_{3, n}\right)
$$

and this finish the proof of Theorem 7.3.

Acknowledgement: This paper condenses results from the author's Ph.D. thesis, supervised by Manoel Lemos, in addition to results obtained later. The author would like to thank Lemos for his exemplary guidance and numerous contributions to this work.

\section{References}

[1] B. Albar, D. Gonçalves and J.L.R. Alfonsín, Detecting minors in matroids through triangles, European J. Combin., Vol. 53 (2016) 50-58.

[2] R.E. Bixby, A simple theorem on 3-connectivity, Linear Algebra Apll. 45 (1982) 123-126.

[3] S. R. Kingan and M. Lemos, A decomposition theorem for binary matroids with no prism minor, Graphs and Combinatorics, Vol. 30, Issue 6, (2014) 1479-1497.

[4] M. Lemos, On 3-connected matroids, Discrete Mathematics. 73 (1989) 273-283.

[5] M. Lemos, On triangle-free 3-connected matroids, Advances in Applied Math. 50 (2013) 75-114. 
[6] M. Lemos, Improving a chain theorem for triangle-free 3-connected matroids, European J. Combin., Vol. 69 (2018) 91-106.

[7] D. Lucas, Weak maps of combinatorial geometries, Trans. Amer. Math. Soc. 206 (1975) 247-279.

[8] D. Mayhew, G. Royle and G. Witthel, The internally 4-connected binary matroids with no $M\left(K_{3,3}\right)$-minor, Men. Amer. Math. Soc. 208 (981) (2010), vi+95 pp.

[9] J.G. Oxley, On 3-connected matroids, Canad. J. Math. 33 (1981) 20-27.

[10] J.G. Oxley, On matroid connectivity, Quart. J. Math. Oxford Ser. 32 (1981) 193-208.

[11] J.G. Oxley, A characterization of certain excluded-minor classes of matroids. European J. Combin. 10 (1989) 275-279.

[12] J.G. Oxley, Matroid Theory, second ed., Oxford University Press, 2011.

[13] J. Oxley, C. Semple and G. Whittlel, The structures of the 3-separations of 3-connected matroids, J. Combin. Theory Ser. B 92 (2004) 257-293.

[14] J. Oxley, S. Pfeil, C. Semple and G. Whittle, Matroids with many small circuits and cocircuits, Advances in Applied Math. 105 (2019) 1-24.

[15] C. Semple and G. Whittle, On representable matroids having neither $U_{2,5^{-}}$nor $U_{3,5}$-minors, Contemp. Math. 197 (1996), 377-386.

[16] P.D. Seymour, Decomposition of regular matroids, J. Combin. Theory Ser. B 28, (1980) 305-359.

[17] W.T. Tutte, Connectivity in matroids, Canad. J. Math. 18 (1966) 13011324. 